\title{
How is an informal transport infrastructure system formed? Towards a spatially explicit conceptual model
}

\author{
Hossein Vahidi ${ }^{1,2^{*}}$ and Wanglin Yan ${ }^{1,3}$
}

\begin{abstract}
The informal transport infrastructure is an inseparable and critical element of the transportation system in that it provides pedestrian accessibility in planned or unplanned environments. Despite this important role, the informal infrastructure is usually neglected in formal studies, plans, reports or maps.

A sophisticated understanding of the different dynamics and mechanisms behind the growth process of the informal infrastructure enables the researchers and practitioners to better plan, conserve and manage open spaces in planned and unplanned environments and helps them predict and manage the growth process of the informal infrastructure in the context of historical cities or informal settlements and model the mutual impacts of infrastructure growth and settlement growth in such areas.

In the absence of a holistic spatially and temporally explicit model in the context of GIScience, this research aims to offer an outlook for some of the most important driving forces and aspects of informal infrastructure formation to establish the principal background for developing a spatially explicit, cognitively plausible conceptual model for future research.

In this sense, this paper presents a critical review to cover a diverse range of topics in the different disciplines of this area and discuss the theoretical issues on the informal infrastructure formation process to explore, analyze and categorize the role of various human individual and collective-level behaviors and various human and environment interactions in emerging of the self-organizing patterns in the informal infrastructure system.
\end{abstract}

Keywords: Informal transport infrastructure, Trail system, Spatio-temporal modeling, Agent-based modeling, Simulation, Complex adaptive system

\section{Introduction}

The Organic Transport Infrastructure (i.e., Organic Infrastructure or Trail) system has unplanned and unstructured organic growth. Consequently, the informal expansion of the infrastructure often has an unstable, unbalanced and inadequate quality that usually suffers from the lack of pavement and formal maintenance.

The organic formation of the human trail system between an origin and a destination node (i.e., point) has a bottom-up nature. Contrary to other modes of transport, pedestrians are not limited to a formal transport

\footnotetext{
* Correspondence: vahidi@sfc.keio.ac.jp

${ }^{1}$ EcoGIS Lab, Graduate School of Media and Governance, Shonan Fujisawa

Campus, Keio University, Fujisawa, Japan

2Lab for Advanced Spatial Analysis, University of British Columbia, Vancouver, Canada

Full list of author information is available at the end of the article
}

infrastructure and can usually walk freely in the environment. The process starts when a track is left behind by the passage of a single pedestrian in the form of physical signs, such as compacted soil or trampled or damaged vegetation. Later, the track develops into a footpath if it has been used frequently by many individuals.

The bottom-up process of organic infrastructure system growth is initiated based on the needs of individuals to provide accessibility in the environment and is influenced by various social, economic and physical factors.

An organic infrastructure can be observed in unplanned environments, such as historic organic textures in rural or urban areas, informal settlements, and natural wild environments.

In the absence of top-down urban design practices, historic districts in rural or urban areas have often been 
expanded from the city center outward as the result of the unplanned spatio-temporal growth process over the course of decades or centuries. However, these old organic structures usually form the backbone and core of the later planned urban texture, and the irregular patterns in their form and morphology may often be partially or fully observed in the current settlements (Fig. 1).

The street networks in historic textures often have organic configurations that emerged as a result of a gradual evolution dynamic over time. The spontaneous and unorganized growth of the aforementioned street networks, referred to as organic networks, forms a dense network of small, narrow and indirect streets that are not organized according to ideal geometric patterns of urban designers but emerged and were redirected according to the daily needs of the residents and included irregular and deformed shapes and intersection angels.

In contrast to the organic historical textures for which the growth process has usually ended for many years, informal settlements are real-life examples of organic growth of the settlements in our era.

UN-Habitat [1] defined an informal settlement (slum) as "a contiguous settlement where the inhabitants are characterized as having inadequate housing and basic services." The housing process in informal settlements often do not operate according to formal laws and regulations and suffer from a lack of formal planning; thus, informal settlements often have irregular housing patterns and are not recognized or addressed by public authorities as an integral or equal part of the formal areas in the city $[1,2]$ (Fig. 2).

Alack of access to proper transport infrastructure is one of the main characteristics of informal settlements that expand in an organic and unplanned dynamic. In the absence of expanded formal transport infrastructure in informal settlements, an informal transport infrastructure network is independently formed by the residents to facilitate connections between significant destinations

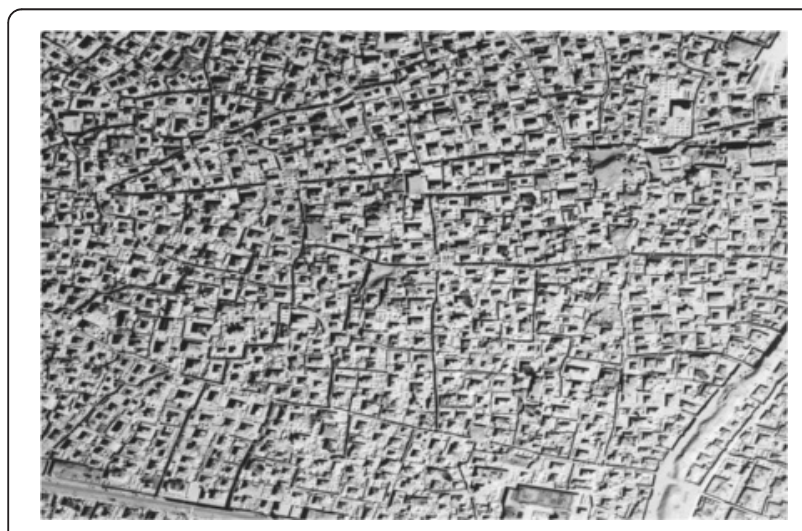

Fig. 1 Spatial pattern of the historical texture in Yazd City, Iran

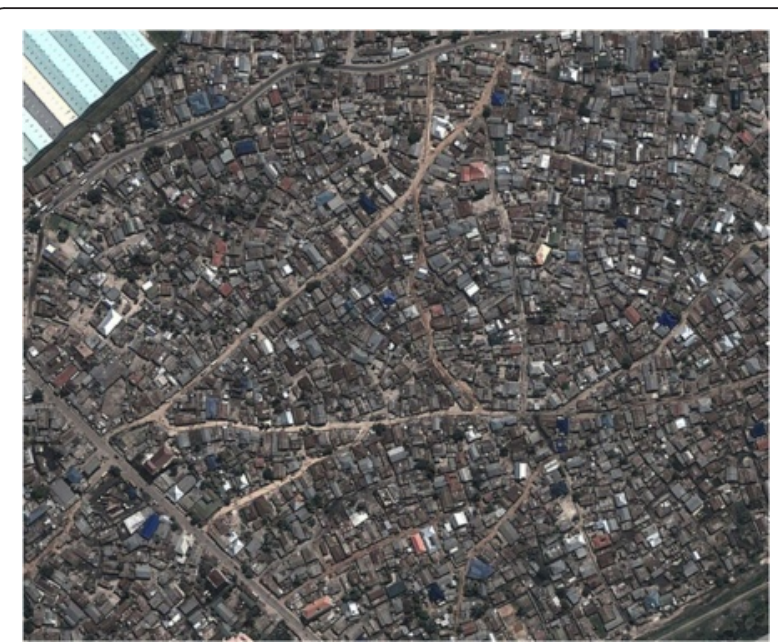

Fig. 2 Spatial pattern of informal settlement in Kipawa Ward, Dar es Salaam, Tanzania (source: Google Earth)

and provides the accessibility to zones and facilities within the settlement (Fig. 3).

The rapid-growth nature of informal settlements usually leads to a chaotic structure with low coverage of organic infrastructure network (footpaths and roads) and narrow and indirect paths that are not organized according to ideal geometric patterns.

An organic transport infrastructure may also be observed in the wild. These trails can be formed in the countryside, wildlife parks, mountains, and beaches by individuals (e.g., tourists, hikers, workers or residents) who walk in the area. Surprisingly, it was observed that these types of unplanned trails are sometimes connected to each other in nature and form long-distance trails with distances of more than one thousand kilometers (Fig. 4).

Thus far, we have talked about the emergence of the organic infrastructure in unplanned environments, but the organic infrastructure formation process can also be observed in formal and planned environments.

A desire path, also known as a desire line, social path or social trail, is a technical term that has been used by urban planners for almost a hundred years to denote a path formed by pedestrians in a formal urban environment [3].

Similar to the formation of the organic infrastructure in unplanned environments, a desire path is normally formed by individuals when there is no formally designed route to pedestrians' destinations or when there is a formal designed route that is inefficient for pedestrians. In other words, if the existing transport network cannot provide the desired accessibility of the pedestrian to his point of interest or an existing formal path to the destination cannot meet the basic principles of human spatial logic in route selection, a desire path may be formed by the individuals. 


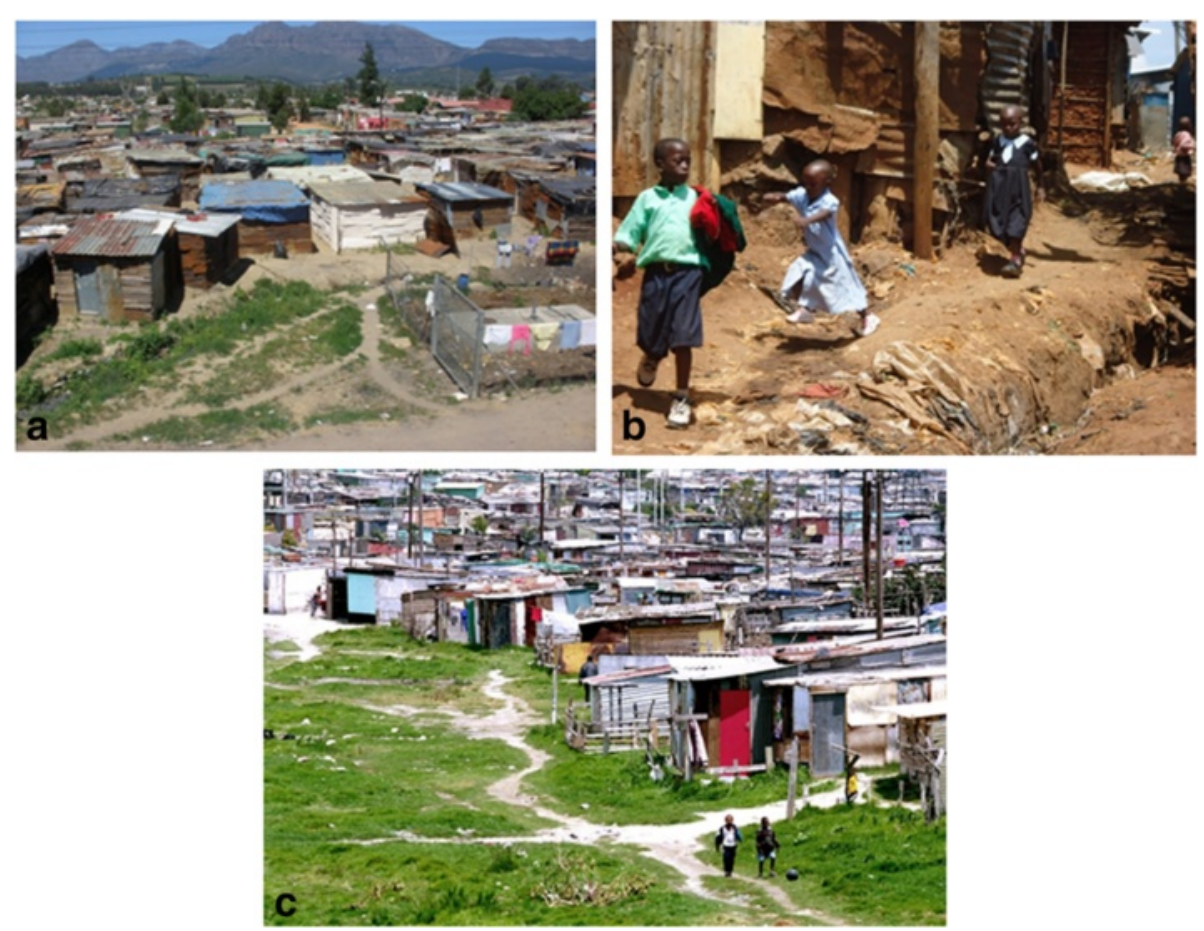

Fig. 3 Informal network of transport infrastructure: a An informal settlement near Cape Town, South Africa (source: reproduced with permission from [71]); b Kibera informal settlement, Nairobi, Kenya (source: reproduced with permission from [72]); c Khayelitsha informal settlement, Cape Town, South Africa (source: reproduced with permission from [73])

In this case, the pedestrians usually go "off-trail" and create their own route to reach the final destination. Over time, a shortcut gradually emerges as a result of footprints in the planned environment. Pedestrians may fully or partially create a desire line on a paved environment. However, as they usually leave behind no markings or less visible markings on the pavement, this type of path is harder to identify $[4,5]$.

Desire lines clearly show the differences between the way of thinking, priorities and preferences of normal people and the planners, which may indicate the fitness of use of the formally planned infrastructure network.

One may see an organic infrastructure at different spatial scales almost anywhere in planned environments, from urban parks or open spaces in the street to university campuses (Fig. 5).

\section{Top-down interventions in organic transport infrastructure system formation}

Although the organic transport infrastructure system has expanded as the result of bottom-up actions and interactions of pedestrians in the environment, a topdown external force may sometimes interfere with this cycle if the track is used frequently by a large number of people for a long time. As the emergence of heavily and frequently used organic transport infrastructure strongly reflects the high demand for formal and efficient accessibility in the neighborhood, this implicit strong social
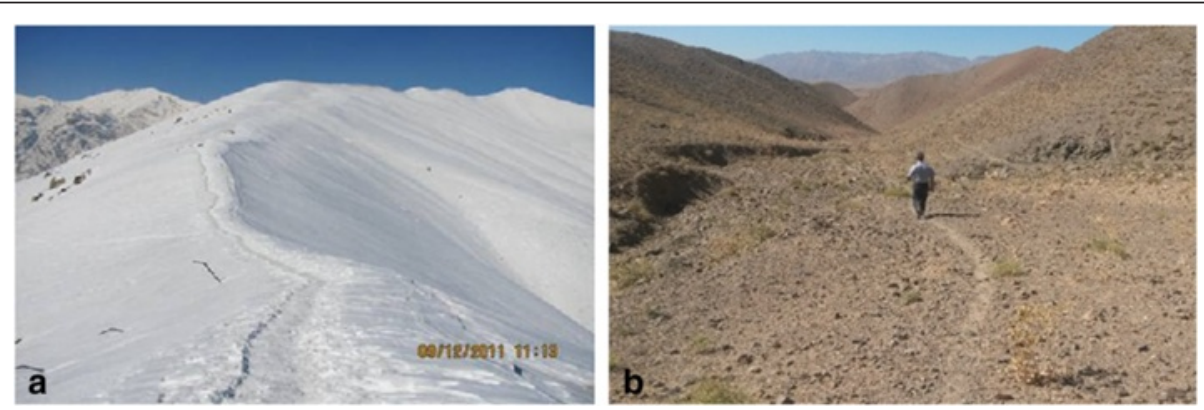

Fig. 4 Organic infrastructure in the wild: a Mountain trail, Darabad, Iran (source: [74]). b Long-distance trail near Khezri Dasht Beyaz, Iran 

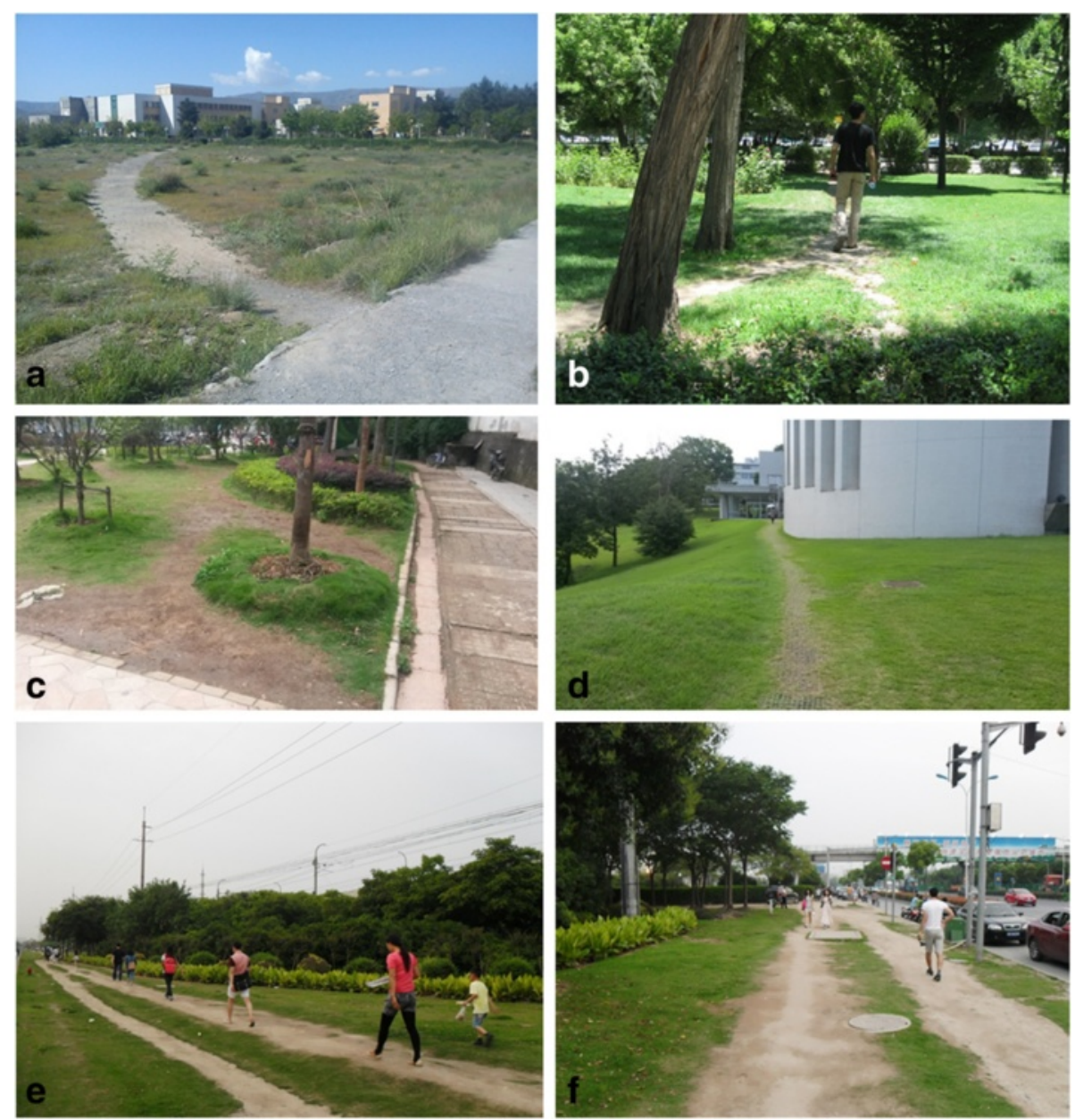

Fig. 5 Organic infrastructure in planned environments: a Ferdowsi University of Mashhad, Mashhad, Iran; b Melat Park, Mashhad, Iran; c Tree lawn, Optics Valley Square, Wuhan, China; d Keio University, Fujisawa, Japan; e \& f Sheshan Station, Shanghai, China

pressure by the track users may encourage the authorities to upgrade or change the pattern and physical condition of the organic infrastructure (Figs. 6 and 7).

For example, because the organic transport network in rural and urban areas with historical textures have been embedded in the texture of the settlement for a long time, the organic infrastructure networks were usually adopted by the authorities later and were upgraded to a formal paved road network. In the case of informal settlements, as the organic network mainly provides the large population of residents with accessibility for homebased or non-home-based trips inside the settlement, the public authorities may sometimes be forced to use top-down intervention to upgrade some major organic paths in the settlement. The top-down upgrade of the trails may also occur in the wild if a large number of people use the trail for mobility within the environment to decrease the degradation of the trail surface and increase the comfort of the visitors and travelers who use the trail. Even in planned environments, an informal trail may sometimes be recognized by the urban management system as a vital element of the planned transportation system when it has been used by a large number of people in their daily lives for a long period of time. In this condition, the official sectors attempts to protect the trail or upgrade it to a paved component of the formal transport infrastructure.

\section{Necessity and importance of understanding and modeling of the trail formation dynamic}

A clear understanding and formulation of the organic trail formation dynamic helps architects and landscape designers design formal paths in open spaces according to human preferences for path selection to prevent the formation of informal shortcuts within the planned environment. Indeed, a well-designed formal infrastructure network that supports all the needs and expectations of the individuals can offer a pleasant experience to pedestrians by providing access to all major destinations of interest in the open space, according to the human 


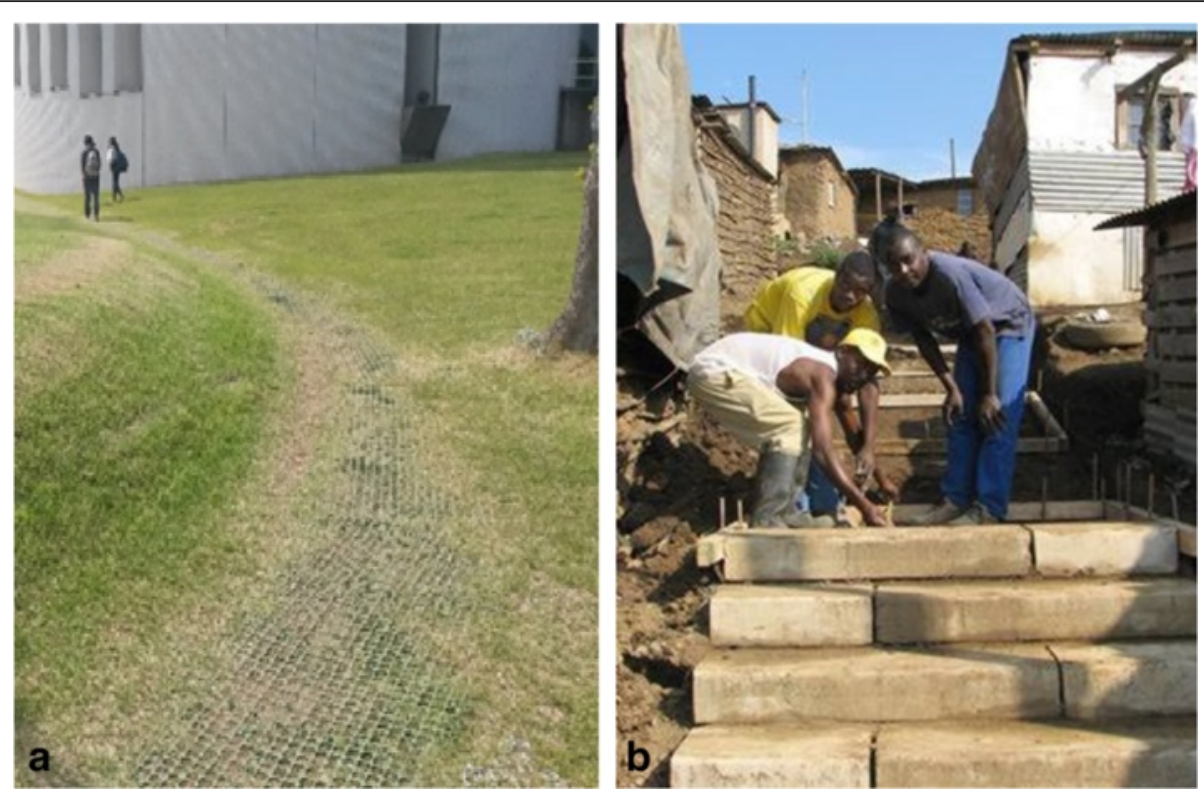

Fig. 6 Trail surface reinforcement and upgrading: a Keio University, Fujisawa, Japan; b Redcliffe informal settlement, eThekwini, South Africa (source: [80])

spatial logic for route selection using a formal, structured, sustainable approach. Additionally, a welldesigned formal infrastructure network may protect the understory vegetation from trampling damage and other types of damage by preventing pedestrians from traveling off-road and creating organic paths; additionally, the environment and beauty of the landscape is protected from fragmentation and destruction (for more details, see [6]).

To understand and model the genesis in historical settlements or historic trails and uncover the socio- economic scenarios behind them, it is needed to gain further insight into the phenomenon of organic trail formation.

In the context of informal settlements, good insight into the different mechanisms and dynamics of organic infrastructure formation may enable urban mangers and experts to predict and control the informal settlement dynamic as organic infrastructure expansion has a considerable role in addressing the informal housing spatial pattern, and consequently, the informal settlement expansion direction (for more details, see [7]).
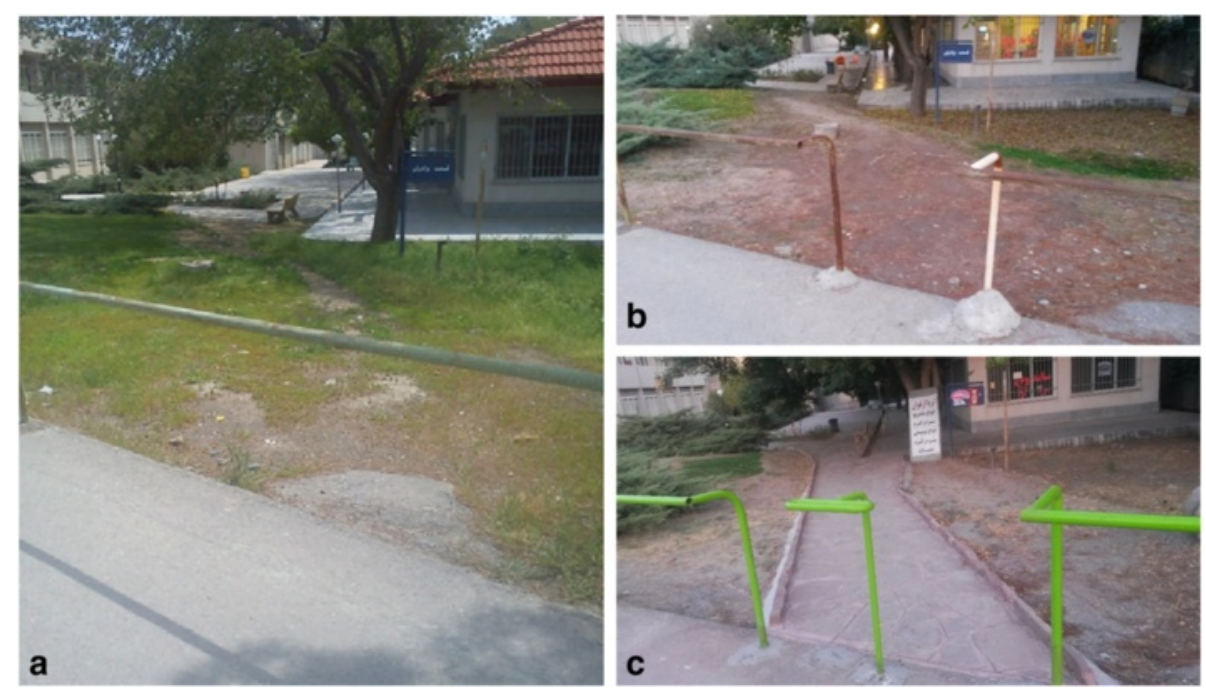

Fig. 7 Gradual adoption and upgrade of a frequently used trail, Ferdowsi University of Mashhad, Mashhad, Iran: a 2012; b 2013; c 2015 
Spatial modeling and the simulation of the organic infrastructure formation process enables one to find an analytical solution for the prediction of organic infrastructure growth spatial patterns according to different social, economic and environmental factors and initial conditions. The outcomes of such a model may help scientists, experts and managers orient, control and even stop the process of unwanted trail network growth by studying the different possible scenarios and analyzing the impact of the different factors in the trail network growth dynamic.

\section{Distinct dynamics of organic trail formation}

Vahidi and Yan [7] categorized the organic trail formation process into two different dynamics. The first dynamic includes a situation where the infrastructure is extended directly as the result of the consolidation of the physical signs of pedestrian locomotion on the environment over time. While the first dynamic of organic infrastructure formation can be observed in both planned and unplanned environments, including informal settlements, they also discussed a second bottom-up infrastructure growth dynamic that is mainly observed in the growth process of informal settlements or the structure of some unplanned historical textures. In this situation, the bottom-up dynamic of infrastructure formation can be considered a function of the settlement housing dynamic. Based on this definition, when a settler constructs a house according to the adopted pattern of housing in the first row of fragments, he usually considers void space to be a path parallel to his house, and this trend has been adopted by other new dwellers in the first row of houses in the fragment. Over time, by forming the second wave of houses in the next row in the fragment, this order is also adopted by the settlers and so on. Thus, over time, the infrastructure penetrates between the houses in the form of void spaces to provide the accessibility of building blocks. Moreover, to connect two parallel, contiguous building rows and provide basic or secondary accessibility, void space has usually been considered between two rows by the dwellers at the appropriate distance.

However, the scope of this study is only limited to study of the first category of the abovementioned dynamics of organic transport infrastructure formation by a group of people; from now on, we will refer to this as informal transportation infrastructure formation, informal infrastructure formation, informal trail formation, informal path formation or simply trail formation in this paper. In this study, our concentration is only on the single-use informal infrastructure that is only used by pedestrians rather than other forms of traffic, such as horses, bicycles or motorcycles.

\section{Research objectives}

Reviewing the existing literature on the organic trail formation growth modeling shows that this topic has not yet received much attention, and further independent studies are needed to clarify the different aspects of the organic infrastructure growth mechanisms. Moreover, almost all of the few aforementioned studies and existing models only concentrate on developing ad-hoc parameters or rules and are mainly developed according to theoretical backgrounds such as mathematics-, physics- or bionicsinspired methodologies without much consideration of human physical, psychological and cognitive behavior.

One of the main problems in the modeling and simulation of pedestrian movement is the lack of human empirical data that usually limits researchers' understanding of the different behaviors and dynamics of the system and makes the task of pedestrian model validation complicated $[8,9]$. Hofmann et al [10] stated that the systematic spatio-temporal knowledge about the different mechanisms of spontaneous structures formation in informal settlements is still absent. The lack of appropriate spatiotemporal data from all steps of the evolution of the system has been made it difficult to understand, model and simulate of the emergent patterns in the informal infrastructure network [11].

Therefore, the majority of the previous studies in this field were devoted to simulating abstract samples without any precedent in the real world or focus on the simulation of a very simplified system that the model often cannot fully include in the real-world formed patterns. Thus, the morphology of the emergent patterns cannot be validated quantitatively (for more details, see [12-14]).

In the absence of a holistic spatially and temporally explicit model that can justify all the emergent spatiotemporal patterns observed in the informal infrastructure network and to fill the existing research gaps, this research aims to provide an outlook on some of the most important driving factors and aspects of informal infrastructure formation by establishing the principal background for developing spatially explicit, cognitively plausible conceptual models for the growth of this geographical phenomenon.

In this sense, a literature review is conducted to collect and analyze all relevant, original articles in the field through an organized and structured search. However, the major goal of this paper is not only to outline the results of the critical literature review on the spatio-temporal modeling of the informal infrastructure formation phenomenon but also provide the grounds for the preparation of a conceptual framework for developing a generic model in the context of Geographical Information Science (GIScience) by considering the findings of the previous studies on this phenomenon in other disciplines, such as psychology and behavioral science, archaeology, physiology, mathematics and physics. Therefore, by crossing the traditional boundaries between academic disciplines, we aim to identify, summarize and categorize the diverse 
conceptual contents of the field that can contribute to spatio-temporal theory development, which is beneficial for developing the holistic models in further work in the area.

\section{Review \\ Self-organization and collective behavior formation in the complex adaptive systems}

A complex adaptive system (CAS) is a special type of complex systems. A CAS is called complex as it is composed of an extensive number of individual interacting components (agents) without any centralized control that generally cannot be derived from or reduced to the summation of the behavior of the individual components of the system as the aggregated behavior of the individuals is not linear. In this term, the adaptive characteristics of CAS describe the capacity of the system for responding to the changes in the environment or among the individual interacting components $[15,16]$.

The system behavior and patterns founds in a CAS emerges from a self-organization process and is not known a priori. Thus, the behavior of a complex system is often referred to as a self-organizing phenomenon. Self-organization is described as a spontaneous process in which a large-scale pattern (including spatial, temporal or spatio-temporal patterns) at the global level of a system originated from the local actions and interactions between the lower-level system components without any internal or external control $[17,18]$.

The global pattern of the self-organizing phenomenon only arises from the processing of information that has been perceived by the components of a system at a local scale without reference to the global information in the emergent pattern $[18,19]$.

The organization of any self-organized phenomenon relies on four fundamental main pillars: (1) positive feedback, (2) negative feedback, (3) random fluctuations, and (4) repeated interactions among individuals $[18,20]$.

The positive feedback loop has been defined as the "system's response to perturbation by reinforcing this perturbation." The reinforcement loop often causes an amplification of specific behavior propagation among the components of a system as a result of the same behavior by the individuals in the neighborhood of each individual in that system.

In contrast with positive feedback, negative feedback dynamics have operated to act against the amplification dynamics to stabilize the collective pattern.

The presence of random fluctuation is another main characteristic of a self-organized phenomenon. The fluctuations in a self-organizing process influenced the system by means of the initiation of the initial perturbation, which is necessary for the beginning of the amplification loop. Some degrees of randomness that existed in the self-organizing phenomena may cause the unpredictability of a particular individual behavior and great flexibility in the system [18]. The roots of this stochastic aspect of the self-organizing phenomenon can be found in that fact that the participating individuals who act in the system do not always respond to the impacted forces in an ideal, deterministic manner.

Self-organizing processes also rely on the repeated interactions among the members of the system. This is the most important driving force for any self-organizing system to produce the complex aggregate patterns at a macroscopic level in the system and is considered the core of any self-organized phenomenon.

The formation of a collective behavior is usually formulated based on simple behavioral rules in biological systems. Hence, the emergence of self-organized adaptive patterns at a global level is the result of the decisions among the members at the local level. This fact compels us to pay more attention to inter-individual interactions and group-choice behaviors because the behaviors and choices of an individual can frequently influence the behaviors and choices of other members in the group [21, 22].

It has been observed that an individual may be more influenced by other members and adopt others' ideas and innovations when they are placed in a social system [22].

Because the innovators have considerable influence over the behavior and choices of follower members in a group, the followers in the group usually pay more attention to others' novel solutions. In this sense, the followers acquiesce to the innovators as they think that the others' decisions may be better [21, 23]. Obtaining social approval is another reason for the emergence of this behavioral conformity and submission to the innovators in a social group. Since an individual often tends to gain social approval from group members in public, they obey the trend and act in the ways to obtain positive evaluations and avoid criticism [22, 24]. Moreover, it has been observed even in the absence of other group members, some individuals are still keen to maintain the trend and confirm others' opinions [25].

Rogers $[26,27]$ stated that innovation diffuses through a certain communication channel from one individual to another in the social system over time. Based on this definition, innovation is judged by the individual based on five intrinsic characteristics. Rogers [27] defined these five innovation characteristics that influence an individual's decision to adopt or reject an innovation as relative advantage, compatibility, complexity or simplicity, trialability and observability that were described in Table 1 .

Using this multi-criteria decision making process, an individual decides whether to adopt or reject an innovation.

Even if an innovation seems very promising and is considered acceptable, few are willing to risk adoption of 
Table 1 Intrinsic innovation characteristics that influence an individual's decision

\begin{tabular}{ll}
\hline Characteristic of innovation & Definition \\
\hline Relative Advantage & Level of superiority of an innovation over the previous generation [27]. \\
Compatibility & Level of compatibility that an innovation has to be assimilated into the life of an individual [77]. \\
Complexity or Simplicity & $\begin{array}{l}\text { The perceived ease of use of the innovation. If the innovation is perceived as complicated or difficult to use, } \\
\text { an individual will not likely adopt it [78]. }\end{array}$ \\
Trialability & $\begin{array}{l}\text { The ease of testing an innovation. If a user is able to try using an innovation, the individual will be more likely } \\
\text { to adopt it [26, 79]. } \\
\text { Observability }\end{array}$ \\
& $\begin{array}{l}\text { The degree to which an innovation is visible to others. An innovation that is more visible for individuals will } \\
\text { drive communication between the peers of the individual and personal networks and will, in turn, make more }\end{array}$ \\
\hline
\end{tabular}

a novel innovation in the initial stage. By contrast, the majority of individuals prefer to wait until early adopters have tested the new idea. Over time, by forming a positive feedback cycle in the system as individuals are convinced of the quality of the innovation, an increasing number of individuals gradually adopt the innovation with a logistical rhythm per time [26, 28, 29] (Fig. 8).

The individuals communicate with each other and exchange their information in the group by means of direct and indirect information transfers. An individual perceives this information and deploys it in the decisionmaking process to determine his next action. On the other hand, it has been frequently observed that the collective integration of individuals' knowledge often leads to efficient behavioral responses by the group in a complex system [18].

An individual may communicate directly with others in the group through different media, ranging from visual or acoustic signals to physical contact. However, other types of individuals, particularly those who are acting in biological systems with many interacting members, transfer their information to other agents indirectly. In indirect means of communication (also called stigmergy), the information is transferred between individuals through alteration or modification in the environment that can be transferred by other agents later. The marking of the environment can be recorded in the environment by different methods, such as physical or chemical marking in the natural environment or virtual tracks in a virtual environment (e.g., internet) [18, 30].

\section{Dynamics and patterns in the formation of an informal infrastructure system}

An informal infrastructure system is formed as the result of the collaboration of all individuals (i.e., agents) in a group of pedestrians (i.e., social crowd or human crowd) without any centralized control in the environment over time. In this term, any single pedestrian is a goaldirected decision-maker that aims to achieve his desired goal by reaching the destination node in the environment of the system. A normal-sighted pedestrian generally extracts information from the environment with his

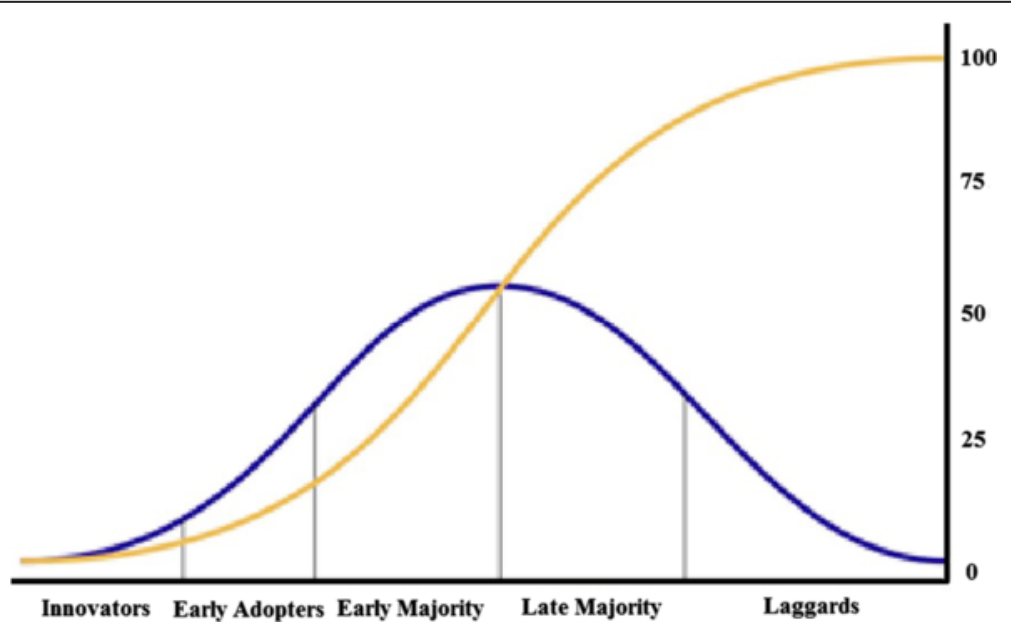

Fig. 8 Diffusion of innovations among members of a social system according to [26]. Distribution of innovation adoption time and cumulative number of innovation adopters are shown in blue and yellow color respectively (modified from [75]) 
visual system and generally communicates with other members in the system in an indirect way. The individual follows several simple rules that characterize each of his responses to the changes in the group and mobility environment. The observation of the evolution of the informal infrastructure system that manifests in the interactions between the pedestrians in the system are critical but the individual pedestrian alone is not critical. Thus, if one or a limited number of pedestrians are omitted from the system, this does not influence the rest of the group and the system. The emerging global-level patterns in the system do not behave in determined ways, but the self-organizing complex patterns in the informal infrastructure system occur as a result of several simple rules. These are discussed in more detail in the following sections.

To understand and model a complex adaptive system, such as the informal infrastructure network, it is not necessary to incorporate all the properties of the system. However, the strategy should focus on extracting and reproducing the pith of the main principles of the system. This can be done by finding the most straightforward explanation in the set of simple behavioral rules for describing the emergent patterns and interactions in the system. Therefore, to understand the collective phenomenon, one can neglect some behaviors and interactions as they may not be necessary or even applicable.

To understand and describe the dynamic of the informal infrastructure network formation, our strategy in this critical review is to simplify the dynamic of the system by breaking down the informal path formation process into simple sub-dynamics, behavioral rules and environmental configurations.

Moreover, one should keep in mind that two dynamics of the human trail system formation and human navigation in the environment are closely interrelated.

Wayfinding and navigation in the environment itself is a complex behavior. The human perceives the information about the environment from multiple sensory complex cues, creates and maintains spatial representations in short-term and long-term spatial memory and applies the created spatial representations to direct navigational behavior [31]. In this term, spatial navigation in humans is performed by using a complex cognitive map to acquire, code, store, recall, and decode spatial information that is perceived from the environment and the application of a hierarchy of navigation strategies that are formulated based on human spatio-temporal thinking skills [32, 33].

Human mobility in the environment is controlled by the human navigation system. Because human locomotion in the environment is the main driving force in the process of informal path formation, the complex behaviors in human navigation are incorporated with the principal rules and sub-dynamics of an informal path.
To decrease the complexity of the system being studied, it is deemed necessary to simplify the system by streamlining the scope of the research by limiting it to the study and review of the principal rules and subdynamics of the informal trail formation process. Thus, in this study, it was assumed that the pedestrian has a general familiarity with the mobility environment and role of spatial memory, and other advanced physiological and cognitive factors in the navigation of the individual in the environment were neglected. In this term, the basic assumption is that the end goal is visible from a single viewpoint or at least at the starting point, and the visual input alone can direct the movement of the pedestrian. Therefore, studying and reviewing the more complicated cases where the final destination is not visible from the starting point for the pedestrian is neglected in this study, as the navigational strategies for human locomotion in a large-scale environment is beyond the scope of this study (for more details, see [34]). Furthermore, for the same reason, other behaviors of the pedestrians and characteristics of the system, such as human collision avoidance behavior, the impact of the number of trail system users and direction of locomotion of the pedestrians on the geometrical parameters and morphology of the trail system and the effects of top-down interventions in the system were not reviewed and analyzed in this study.

To provide a clear understanding of the different behaviors and characteristics of the informal infrastructure system components and to build a holistic framework for developing a robust conceptual model(s) for the spatio-temporal modeling of informal infrastructure formation phenomena for future studies, the results of the critical literature review on previous studies were summarized, categorized and analyzed. Furthermore, due to the lack of sufficient previous contributions to the study of the different driving forces and dynamics in the process of the informal infrastructure formation as well as the common lack of empirical data for pedestrian movement behavior, an integration of the knowledge of the authors that was grounded in the long-term observations of informal infrastructure systems was attempted, with the result of the critical review for the discussions establishing good groundwork for conducting future studies in this area.

In the following sections, we first consider a flat, obstacle-free and uniform (i.e., unvarying in terms of the walking preferences of pedestrian for traveling on the environment) environment and only focus on understanding and reviewing the collective behavior of single individuals who form the trail system in the Simple and Compound Topology of the origin and destination node(s) as well as the emergent large-scale, self-organized patterns in trail system. Then, the process of trail formation on complex 
terrain will be explained, reviewed and discussed. Finally, the life span of the informal infrastructure will be briefly discussed to summarize and identify the open and noteworthy issues for future study.

\section{Formation of the human trail system in a simple topology of origin and destination node}

Visual perception is the primary basic channel of any pedestrian to acquire data on static and moving objects in close and distant environments. These data are utilized to manage human locomotion by controlling the dynamic stability, forming the basic patterns for the various environments at the local level, starting and finishing locomotion as and when required, and including efficient locomotion guidance and route planning to destinations at the global level (For more details see [34, 35]).

Wayfinding and route planning are tasks that are performed as an individual moves from one location to another [36]. Passini [37] defines wayfinding as spatial problem solving that consists of information processing, decision making, and decision execution. In another definition, wayfinding was defined as the ability for humans to recognize locations and approaching destinations, both cognitively and behaviorally [38, 39].

The human decision making process during the wayfinding process is carried out in almost an automatic, rapid and unconscious approach.

The pedestrian as a decision maker considers the locomotion process as a sequence of choices over time, in which the pedestrian decides where he will be at the next time step in the space around his present position [40].

Thus, wayfinding and route planning are procedures based on a dynamic spatial-temporal decision-making process in which an individual has to make immediate location-based decisions [41].

To process the different dynamics of human mobility in the environment, we first consider a "simple topology" in that there is only one origin node (i.e., start point) and one destination node (i.e., end point) in the set of nodes for all pedestrians in the system (Fig. 9).

All pedestrians, regardless of age, gender or level of mobility or skill are motivated to reach to their destinations successfully. However, from the perspective of a pedestrian, the concept of success might have different definitions depending on the mobility environment and personal attributes of the individual. In these terms, human spatial logic for route selection navigates an individual from the origin to the destination generally in favor of optimizing a certain utility function (i.e., cost function) to ensure the expected success. The cost of human movement in the space can be measured in several ways, such as energy and time. Among the different studies published on this topic, we can use [42-51] as examples.

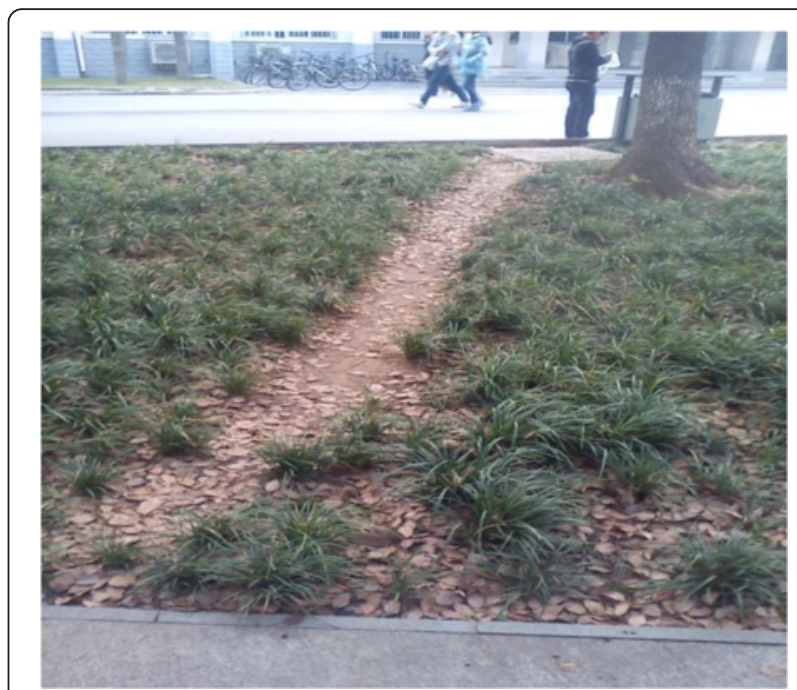

Fig. 9 A short trail formed in a simple topology of the origin and destination node, China University of Geosciences, Wuhan, China

In a simple environmental configuration, to optimize the locomotion utility function, the pedestrian reaches the destination node so as to minimize the metabolic energy (i.e., metabolic cost) of his mobility roughly according to the visually perceived information, which is acquired from the environment, mainly at a local scale.

In practice, if the distance between the start and end points is relatively long, an individual solves the global scale optimization problem of finding the minimum energy path by breaking the problem into several spatial intervals and optimizing the consumed energy at the local level. Therefore, the pedestrian employs the path selection rulesets for the smaller spatial intervals by considering some temporary destinations between the start and end points over time.

In an ideal condition, and if the human movement surface is flat, obstacle-free and uniform, the problem of finding a minimum-energy expenditure path for an individual is considered equal to the problem of finding the shortest path from the origin to the destination node.

However, as the mobility of the pedestrian is mostly controlled by vision in practice, an individual might detour slightly from the Euclidean shortest path connecting two given nodes (i.e., beeline path), as vision-based navigation is inherently an error-prone method.

Moreover, in the context of cognitive sciences and behavioral decision theory, past studies showed that in realistic conditions, the decision-making process for wayfinding is not optimized strictly based on mathematical or economic factors but also on other behavioral and cognitive factors that are part of this process $[41,52]$.

The impact of other above-mentioned factors besides the mobility skills of the different pedestrians and the environmental condition are usually influenced by the 
step-by-step choices of an individual. In this term, the spatial fluctuations (i.e., biases) of the beeline path emerge in the locomotion of a pedestrian. As a result, the spatial solution of an individual (i.e., the selected path) usually has some degree of detour from the ideal Euclidean shortest path. Furthermore, the impact of different factors on the decision of the different pedestrians with the same goals and conditions might be slightly different, so even the solution of two pedestrians for the same energy-expenditure optimization problem may not be exactly the same.

A pedestrian stores his solution in the environment by altering the background of the format of physical markings through his locomotion from the origin to the destination.

In other words, the spatial information for the adopted solution for locomotion from the origin to the destination is recorded instantaneously in the environment through the pedestrian's footprint impact on the environment in terms of compacting or eroding the underlying soil or snow and damaging or trampling the ground vegetation. In this way, every individual involuntarily shares his solution for the system by using an indirect communication channel (i.e., stigmergy) by means of modification in the environment's properties.

In the process of finding the minimum-energy expenditure path, an individual may give a more privilege to adopt others' solutions (i.e., minimum-energy expenditure path) if he is more convinced that there is unanimity in the competence of the solution between several group members. Therefore, when an individual sees other footprints in his vicinity in the direction of the destination, he may decide to take the risk of deviation somewhat from his own path to pass over that area and adopt the track in the intersection of the solution sets generated by other individuals.

Because an area has been selected frequently by different pedestrians, it has been consolidated properly and has a smoother surface. This fact causes the equilibrium of the system to be off-balance in terms of the homogeneity of the environment. As the ease and comfort of mobility in better trodden areas are much higher in comparison with unprocessed areas in the environment, more pedestrians are willing to travel through these areas. Furthermore, by passing from common areas (i.e., frequently used areas for travel), the chance of having social interactions with other track users is increased for an individual. In this sense, making contact with other pedestrians nearby and having social contact ensures that an individual conforms to the majority's solution (which he usually considers the best solution). In addition, this social interaction increases the positive physiological feeling of safety in an individual, especially when the destination is far and the environment is unknown.
Therefore, the characteristics of well-processed locations in the environment increases the desirability of these locations for individuals over unprocessed locations as less of an energy expenditure is needed for walking through these areas as well as a greater chance of social interaction in these areas.

In this term, the concept of "success" in the locomotion of an individual from the origin to the destination node carries a dual meaning. In this condition, the minimization of the travel distance and the maximization of the comfort and simplicity of the mobility are considered by the pedestrian. Therefore, in this case, the spatial problem of finding optimal-energy expenditure paths transforms into a broader problem of finding the most comfortable path for locomotion.

An individual judges the selected solutions of the first generation of the pedestrians in the systems according to the major criteria that were mentioned in. In the decision making process, he examines the relative advantage of the existing innovations of predecessors according to the factors of proximity, comfort and level of social interaction. If the innovation can direct him to the final destination successfully, he may find the solution compatible. If the predecessors' solution is not far from his spatial logic and the pedestrian can adopt it easily with his mobility skills and other factors, he considers the innovation simply as an adoptable solution. The pedestrian considers an existing track a testable innovation if the environmental condition and his psychological and physical characteristics allow him to move over the track. Well-trodden spots, particularly if they are placed in the proximity of pedestrians, attract more attention as they are more observable for the pedestrian in the environment.

Gradually, the created positive feedback loop in the system is strengthened in favor of selecting the frequently used areas, and the unprocessed areas in the environment are much more likely to be ignored as the result of a formed negative feedback loop. Thus, over time, by forming a reinforcement loop in the system, more individuals gradually tend to use the consolidated areas in their locomotion process from the origin to the destination.

Since the positive feedback cycle has been formed, the motivation for new pedestrians to travel on well-trodden paths increases sharply, and more individuals adopt the group collective solution to the spatial problem of finding the optimal path. Through the diffusion of the innovations of the pioneer pedestrians over time, a physical macroscopic pattern gradually emerges in the environment in the form of a trail as the result of a selforganizing process. The emergent trail pattern at the global level is the result of a collective strategy of the social group in finding the optimal path from origin to destination. In this context, the group tends to find a path among the different alternatives that can satisfy two 
constraints: (1) the path has a shortest possible length and (2) the path offers the maximum possible ease of travel. This multi-criteria decision-making problem has to be formulated by compromising between the original solution of the individual and the predecessors' solutions that were recorded in the environment.

We simply illustrated the workflow of the trail formation process in the simple topology of the origin and destination node in Fig. 10 with the UML 2.0 activity diagram.

This diagram displays the sequence of activities in the environment from the starting point to the end point for the pedestrian (i.e., agent), detailing the many decision paths that exist in the progression of events contained in the trail formation activity.
Formation of the human trail system in the compound topology of the origin and destination nodes

The trail system structure may sometimes emerge in the more complicated topology of the nodes than a simple topology of the origin and destination nodes. In this study, a combination of the topology of the nodes are referred to as the "Compound Topology" of the origin and destination nodes where there are more than two nodes (i.e., one start and one end point) in the set of origin and destination nodes (Fig. 11).

To our knowledge, there are only two independent principal studies that aim to understand the dynamic of human trail system formation in the natural environment.

Helbing et al. [12, 53, 54] developed an innovative simulation model to reproduce the observed trail system

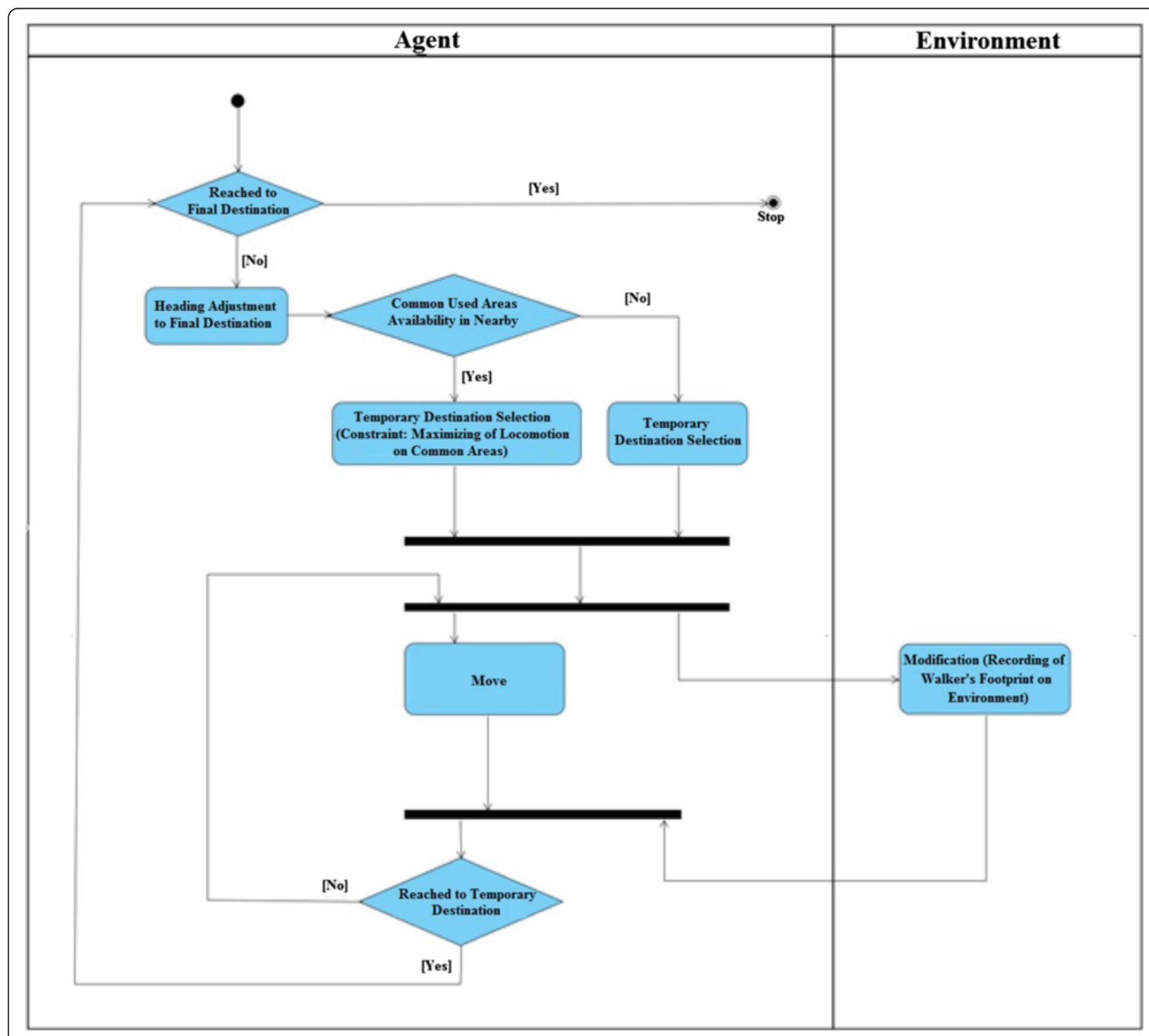

Fig. 10 UML 2.0 activity diagram for trail formation process workflow in the simple topology of the origin and destination node 


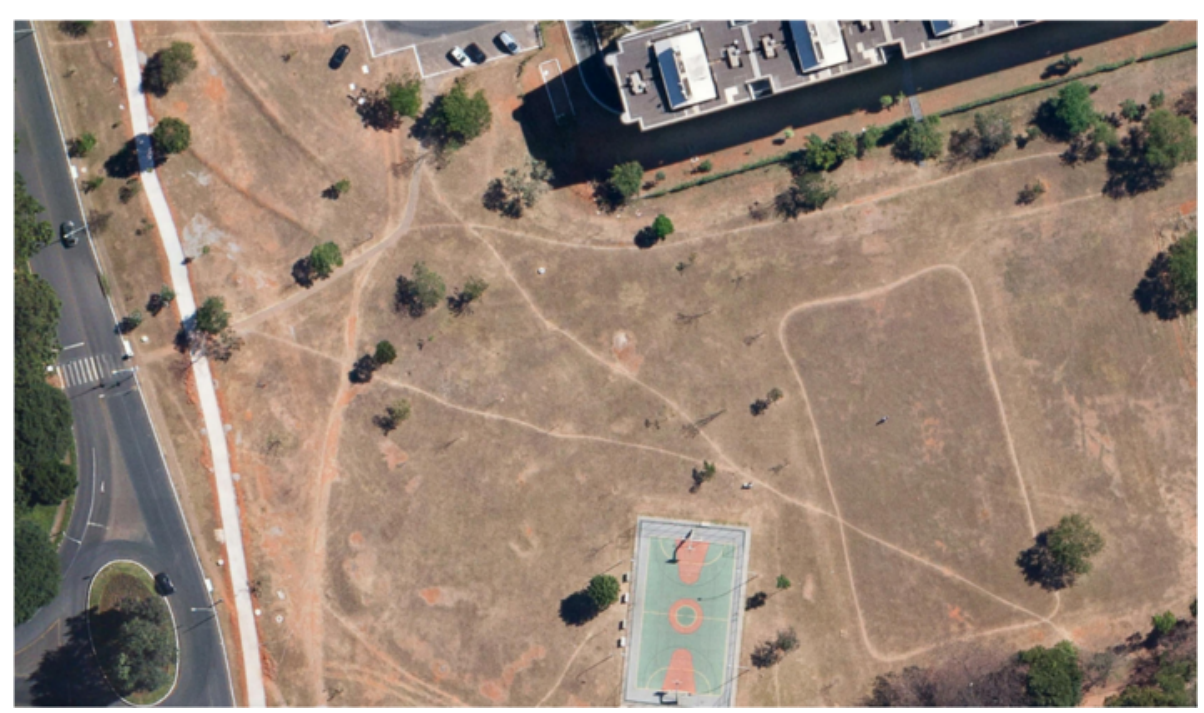

Fig. 11 Y-shaped trail system formed in the compound topology of the origin and destination nodes, Norte 106/107, Brasília, Brazil (source: Google Earth)

patterns in the Compound Topology of the origin and destination nodes. For a simulation of the trail formation process, they adopted a physics-inspired approach based on active walker models that were formerly proposed and developed in the 1990s by groups of researchers in the field of theoretical physics. However, to describe and justify the dynamics of human trail formation, they attempted to consider certain psychological principles of human behavior.

To interpret the dynamic of the formation of the selforganizing trail patterns in the compound topology of nodes, they used an explanation analogous to the one we adopted for the dynamic of the trail system formation in a simple topology of nodes: the bottom-up and evolutionary nature of trail formation and the inherent tendency of individuals to optimize both the length of their trip and ease of travel. According to their proposed conceptual model, at the beginning, the pedestrians use direct ways between the nodes. However, according to their explanation, over time, the pedestrians may be attracted to existing trails in the environment. In this term, they stated that if the attractiveness of the existing trails is significant enough for pedestrians to travel, over time, a physical structure referred to as the "Minimal Way System" (MWS) is formed by the pedestrians that represents the shortest way system that connects all nodes in the system.

However, they stated that the trail system evolution normally stopped before reaching this state, as pedestrians do not like taking a large number of detours from the direct path (beeline path). As a result, they stated that a compromised trail system, which they referred to as a "Minimal Detour System" (MDS) is formed in the system. Figure 12 depicts the results of the simulation performed by Helbing et al. to model the trail formation between the three nodes according to the different trail attractiveness values. If the trail attractiveness value in this model is low, the "Direct Way System" (DWS)
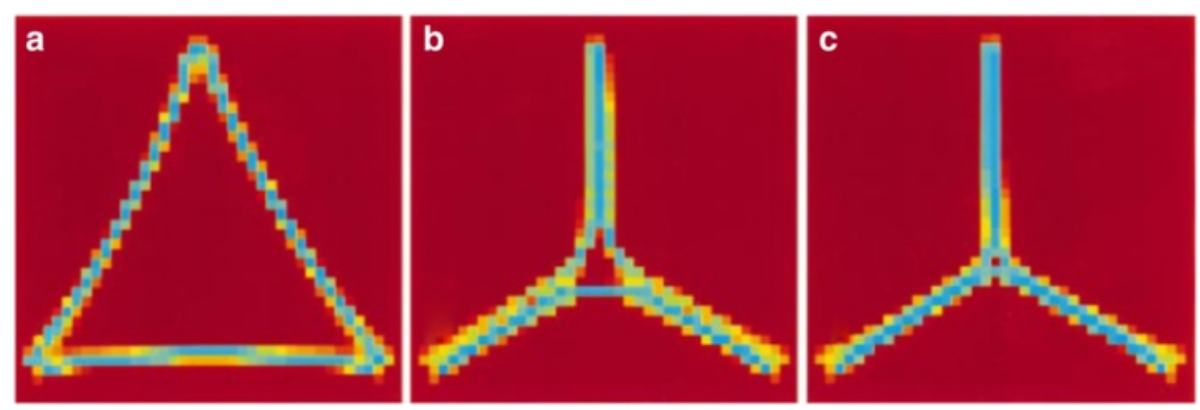

Fig. 12 Simulation result of the Helbing et al. model of the trail formation between three nodes according to different trail attractiveness values. a DWS, b MDS, and c MWS (source: Adapted by permission from Macmillan Publishers Ltd: [12], copyright (1997)) 
structure is formed between the three nodes (a). If this value is selected with a very large value in the model, the MWS structure emerges (c). Otherwise, a compromised structure for the MDS is formed between the nodes (b).

In another approach, Goldstone et al. [22] and Goldstone and Robert [55] studied the nature of collective behavior of human crowds in forming trail system patterns in the compound topology of origin and destination nodes. To study this phenomenon, they developed a software platform for tracking the virtual movements of the participants between several predefined nodes (i.e., destination points) in their virtual experiment. In this situation, a participant used the keyboard to navigate a virtual pedestrian in a virtual system to simulate the movement behavior of a real person in the real world. In this multi-user network game, a participant can observe his moment-by-moment movement as well as the movement tracks of other participants in the system. The designed virtual environment in this system was homogeneous, flat, obstacle-free and has a uniform surface that was constructed from a $150 \times 100$ grid to simulate the walking environment.

To model the process of change in the ground conditions as the result of human walking, the experiment considers a changing value for each cell in the grid. To model the walking comfort level in the real environment, the cell's cost in the system decreased as a function of the number of times it had been virtually trampled. To modeling the human energy expenditure amount, the participants were asked to virtually walk from their origin to the destination so as to earn the highest number of points possible.

In this system, the participant's earned bonus point total was shown on the participant's screen. The participants were told that they would gain a certain number of bonus points if they reach a destination and lose a certain amount of bonus points for each step they took. The popularity of a cell was estimated and illustrated for the participants by the cell's brightness scale, so the brightness of a cell is proportional to the number of the users who selected that cell for travel. Therefore, if a cell was selected by a large number of participants in this example, it appears brighter on the screen.

In this experiment, each node may serve as both the origin and destination, and the designated algorithm was distributed the trips uniformly between the nodes.

In total, eight different compound topologies of nodes were examined in this experiment. Figure 13 illustrates the result of the Goldstone et al. experiment. This result shows that some degrees of deviation from the beeline path (i.e., direct way - the Euclidean shortest path between two nodes) can be observed in the formed trails in the system.

To explain the emergent patterns in this experiment, they adopted the "Minimum Steiner Tree" (MST) concept from the combinatorial optimization in applied mathematics.

The MST, named after Jakob Steiner, describes the way to formulate the minimum interconnection of a given set of vertices in a graph. In the other words, the MST is defined as a tree that connects the set of vertices with the shortest possible total lengths of the network. The MST-finding problem is very close to the minimum spanning tree problem. However, in contrast with minimum spanning tree problem, in the MST problem, one may add the extra new points (i.e., nodes, vertices), or so-called Steiner points (i.e., Steiner nodes, Steiner vertices), to the system to reduce the total length of the spanning tree $[56,57]$. A Euclidean Steiner tree has two main characteristics [58]:

1. The Steiner point in a Steiner tree must have a degree of three (i.e., the Steiner point is connected exactly to three other points).

2. A three-edge incident to a Steiner point forms three $120^{\circ}$ angles.

Figure 14 shows the MST structure pattern for three and four points.

Goldstone et al. [22] stated that although the deviations in the formed trail systems in their experiment do not generally form the ideal MST structures, the deviations usually move in the direction of forming MST structures. They claimed that the individuals tend to form the approximate MST-like trail systems to optimize the total length of the trail system. However, they stated that the deviation toward an MST structure is not always observable in the trail systems, as there is no guarantee that a group was always able to find the aforementioned semi-optimal configurations.

In this term, to refer to the semi-optimal trail structure and address the deviation in the trail system for the compound topology of nodes, they employed the term of "pro-Steiner" structure and deviation.

Up to now, we have reviewed the findings of the Goldstone et al. experiment. However, it is obvious that the virtual nature of their experiment may impair the realism of the resulting behaviors. This experiment was conducted with the participation of a limited number of users who used their computers to mark their decisions (i.e., their selected route) on a screen in a short period of time. Moreover, in contrast with the real world, the participant of this study has global knowledge of the environment and emergent trail patterns as they see all emerging patterns online. Therefore, it is not an easy task to over-generalize the results of this study to the realistic topologies of nodes in the real world.

However, as is observable, generally speaking, one may say that if the topology of nodes has a smaller-scale 


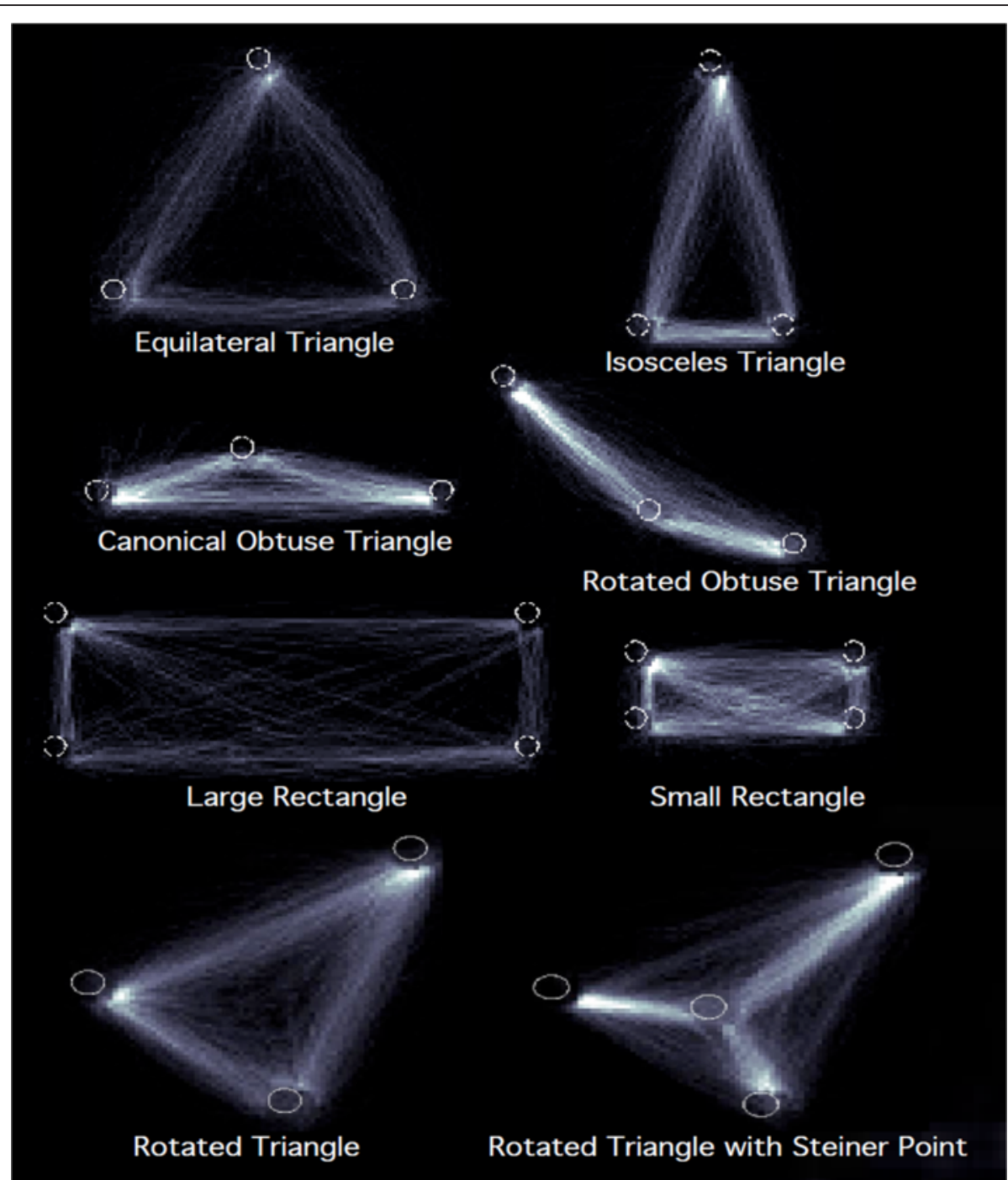

Fig. 13 Emergent trail system patterns between eight different topologies of nodes in Goldstone et al. experiment (source: ( $[2006]$ IEEE. Reprinted, with permission, from [22])

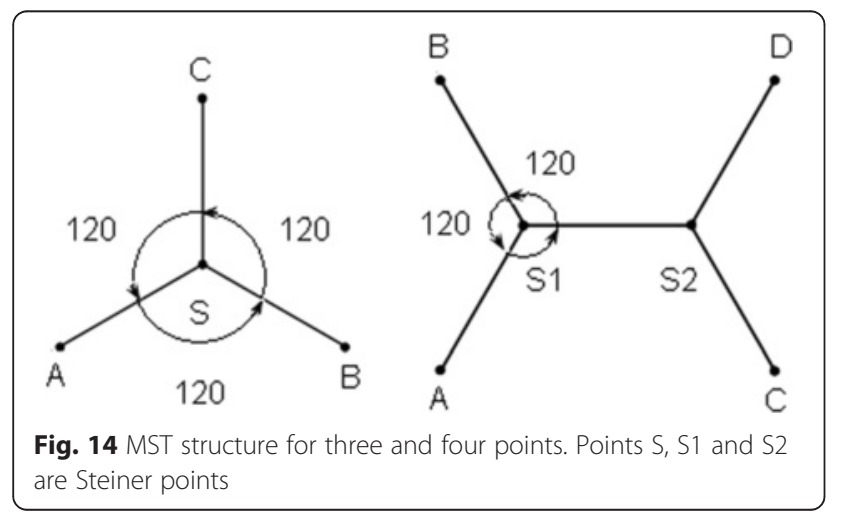

factor and vertex angle size, the probability of overlapping and merging the beeline tracks and extending the longer common paths are increased.

The comparison of two theoretical studies by Helbing et al. and Goldstone et al. shows that although they adopted different terminologies for referring to the emergent patterns and deviations in the formation of the trail systems, the proposed core concepts both adopted for justifying the emergent trail patterns seem very similar.

First of all, the MWS concept used by Helbing et al. fundamentally has same function as the ideal MST structure that was argued by Goldstone et al., as both terms discuss the same notion: the minimum total interconnect for a given set of nodes.

Furthermore, in both studies, the authors argued that the trail system formation dynamic usually stops before reaching the optimal structure (i.e., MWS or MST 
structure). In this context, both studies claimed that the evolution of the trail system usually stops at a semi-optimal structure that they refer to as a MDS and "pro-Steiner" structure, respectively. However, despite the similarities between the findings of two studies, some differences are also observable between the Helbing et al. and Goldstone et al. perspectives.

As mentioned before, Helbing et al. stated that the compromising mechanism by human groups in the context of minimizing the individuals' path length and maximizing the pedestrians' ease of travel and comfort has a main role in the formation of the MDS. However, Goldstone et al. place a greater emphasis on the group's motivation in minimizing the total length of the trail system and using the common tracks as the main driving force of the collective path formation. In this sense, they believe that the individuals move in the direction of forming of an approximate minimal total length trail system, the so-called "pro-Steiner" structure.

Helbing et al. claimed that the trail system evolution "usually" stops before reaching the MWS structure, but they have not explicitly rejected the possibility of forming the MWS in the compound topology of the nodes in their study.

However, we believe that reaching the minimal total length trail system (i.e., MWS or MST structure) is not the goal of the pedestrians during the formation of the informal trail system in the compound topology of nodes.

Helbing et al. did not introduce any realistic trail samples with the MWS to support their claim, and their study was only limited to the results of several computer-based simulations.

In contrast to this claim, the results of Goldstone et al. clearly indicated that the deviations that emerged in the formed trail patterns of their experiment do not generally form ideal MST structures. Furthermore, one should keep in mind that in the process of informal path formation, the basic goal of any pedestrian is to maximize his self-interest during walking (i.e., minimize his own mobility length and maximize the ease of travel). In this situation, the individual's behavior in the process of informal path formation can usually be considered selfish behavior. Therefore, it is expected that a selfish individual only thinks about minimizing his own interest between his origin and destination instead of considering other interests and forming the minimal total length trail system (i.e., MWS or MST structure) between all the nodes in the compound topology of origin and destination nodes, as using the minimal total length trail system usually results in a large detour from the shortest path between the origin and destination.

The lack of global knowledge about the general pattern of an individual as well as the computation complexity of the MST problem is another reason for our claim. The MST problem is categorized as a NP-complete problem, and it was proven that as the number of vertices in the network increases, the computation complexity for finding the MST increases significantly [57].

Pedestrians usually do not have clear global knowledge of the environment and the topology of the origin and destination nodes in the large scale environment. Therefore, one may say that an individual was not usually able to obtain sufficient data (directly or indirectly) from the environment to solve the MST problem, particularly when an individual has to solve it with a large-scale topology of nodes.

Furthermore, as mentioned before, the pedestrian exhibits goal-oriented behavior. Hence, to solve the complex optimization problem of finding the MST structure (through an exact method or heuristic approach), it is expected that the goal of finding the minimum total interconnect (or at least finding a sub-optimal solution) for a given set of nodes must be determined explicitly (or at least implicitly) as a strategic target. However, this target is not included among the goals of an ordinary pedestrian.

We believe that the formation mechanism of trail system patterns in a flat, obstacle-free and uniform environment is the same as the trail system formation mechanism in a simple topology of nodes. In this sense, a DWS is formed in the initial stages of informal trail formation as the result of a locomotion distance minimization goal of pedestrians. Over time, with the emergence of common tracks in the environment, a pedestrian will tend to use common tracks to increase his convictions and ease of travel. However, as minimizing the energy expenditure (i.e., minimizing the locomotion distance) is a major constraint, the individual usually is not interested in making a large detour from the beeline path (i.e., shortest path), so he tries to find the balance between these two main goals (i.e., minimization of the locomotion distance and maximization of conviction and ease of travel). To find a compromise in the flat, obstacle-free and uniform environment, a pedestrian tries to determine the answer of the following questions in the continuous process of route planning from origin to destination:

- To what degree is the candidate common track consolidated?

- What is the distance between the individual and the candidate common track?

- How much of a detour from the beeline path is needed if he wants to take the candidate track?

By consolidation of the compromised trail system, a balanced structure is forming in the trail system.

In this study, we refer to this structure as the "Balanced Way System" (BWS) instead of the previous adopted 
terms of MDS or "pro-Steiner" structure in the past studies; we stated that the optimization of the total structure of the trail pattern is not the goal of any single pedestrian.

Figure 15 illustrates the UML 2.0 timing diagram for a schematic discrete depiction of the lifeline of the evolution in the spatial structure of the informal infrastructure in a compound topology of origin and destination nodes.

The lifetime of each state is the function of various parameters, such as the number of the trail users and frequency of trips, the physical characteristics of the surface, and weathering conditions in the system.

\section{Formation of the human trail system in complex terrain}

Heretofore, the basic assumption in the previous sections was that the environment is flat, obstacle-free and uniform. In contrast to this idealistic assumption, in reality, a walker usually encounters more complex terrain configurations.

As we described earlier, the human spatial logic for route selection navigates an individual from the origin to the destination generally in favor of optimizing a certain utility function to ensure his expected success. The concept of success, as mentioned earlier, includes much more than simply finding the shortest path between the origin and destination and may include a broader domain such as mobility comfort, safety and efficiency, or experiencing pleasure during travel.

Therefore, to explain the dynamic of the trail system formation in sloped, obstructed and non-uniform environments, we extended the concept of the "optimum comfort path" for locomotion that was described before to a broader domain to meet all other physical, social and environmental factors for addressing the path with minimum human dissatisfaction.

Therefore, in this sense, the typical problem of finding the minimum energy expenditure path will be altered due to the problem of finding a compromise solution between the individual and predecessor solution that meets the common physiological, social and economic constraints of the group simultaneously and ensures the expected success of all factors.

In this sense, the minimization of the total travelled distance and establishing the DWS still remains the main priority of pedestrians at the first stages of trail evolution. However, if a pedestrian faces sharp elevation changes, physical or non-physical obstacles, or nonuniformity in the environment during his travel, he may decide to detour from the shortest Euclidian path to avoid steep slopes and impassable obstacles or travel in more pleasant areas.

The pedestrian roughly weighs different parameters based on his priority list and ranks the locations in the environment within the domain of his vision span based on the suitability of the points from his point of view. Therefore, the pedestrian generally attempts to select his path according to his inclination (e.g., passing through shaded areas in the summer or walking on hard and dry surfaces) and disinclination (e.g., not encountering steep slopes or impassable obstacles) with the shortest possible length of the trail to optimize the energy expenditure of the path, according to other criteria.

Over time, by the formation of the common tracks in the open space, a pedestrian will tend to pass over the common tracks to increase the level of his mobility convenience and ease of travel and the compromise solution of the BWS forms.

In the following sections, we discuss the principles of the adopted strategies of the individual in the process of the informal trail formation in a complex terrain where the terrain is sloped, obstructed or has non-uniform conditions.

Formation of the trail system in a sloped environment A considerable number of studies have been carried out to find optimal paths on sloped and rugged terrains based on different theoretical background and approaches for energy, time, and other types of optimization (e.g., [14, 46, 49, 50, 59-61]). The review shows

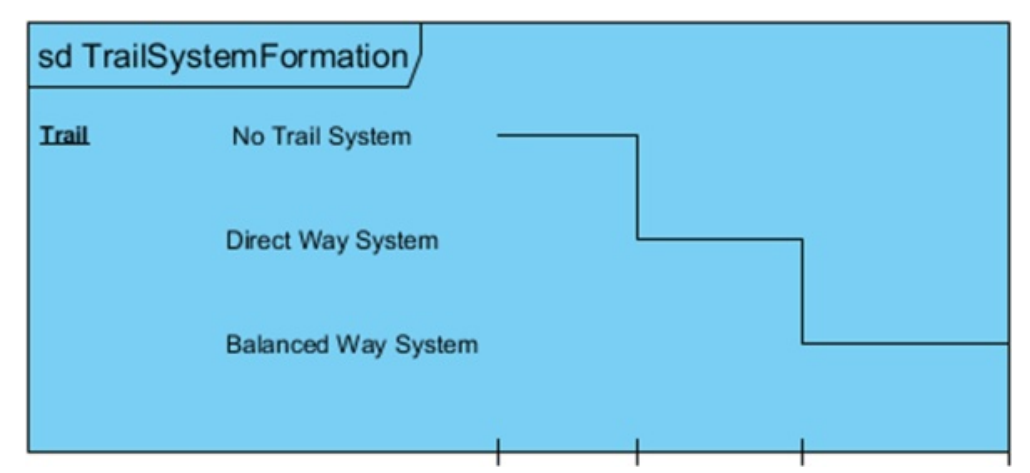

Fig. 15 UML 2.0 timing diagram for evolution in lifeline of an informal infrastructure spatial structure in the compound topology of origin and destination nodes 
that the existing literature mostly focused on studying human movement on the sloped terrain rather than the path-formation process, and previous studies mostly concentrated on suggesting optimal path-finding algorithms for a walker who is ascending or descending a mountain or traversing rugged terrain according to an ad-hoc parameter (i.e., optimization of metabolic energy travel time, etc.).

Reviewing the few studies $([13,14,49])$ that oriented or aimed to model the trail formation process or reproduce the trial patterns emerging on sloped terrains reveals that all of the proposed models were more reliant on theoretical backgrounds, such as mathematics and physics-inspired optimization methodologies, and contributed little attention to human physical, psychological and cognitive behaviors. In this context, Gilks and Hague [13] extended the active walker model to address the formation of trails on gradients. However, the other contributions [14, 49] are reliant on optimization methodologies for reproducing the emerged trail patterns that are observed in rugged environments, and they do not include the cost (i.e., comfort) of using formed trails in the cost estimation of their optimization algorithm. Moreover, the majority of previous studies were performed only on some simple geometric non-realistic shapes that have a constant inclination angles, and broader problems in the real world were neglected (Fig. 16).

In this section, we aim to review the important physical, psychological and cognitive behaviors of humans in the process of informal infrastructure formation on sloped terrains.

Walkers usually prefer to move on flat surfaces and travel parallel to a contour line, as a rugged terrain and change in the elevation of the surface (i.e., slope) and the direction that a slope faces (i.e., aspect) are likely to lead to discomfort as the result of the instability, speed reduction and fatigue caused by the consumption of greater metabolic energy.

When one walks uphill, more energy is needed to overcome the gravity force to carry body mass than walking downhill. By walking downhill, the body is carried down the slope due to the gravitational pull, and only energy has to be consumed to brake in order to prevent falling.

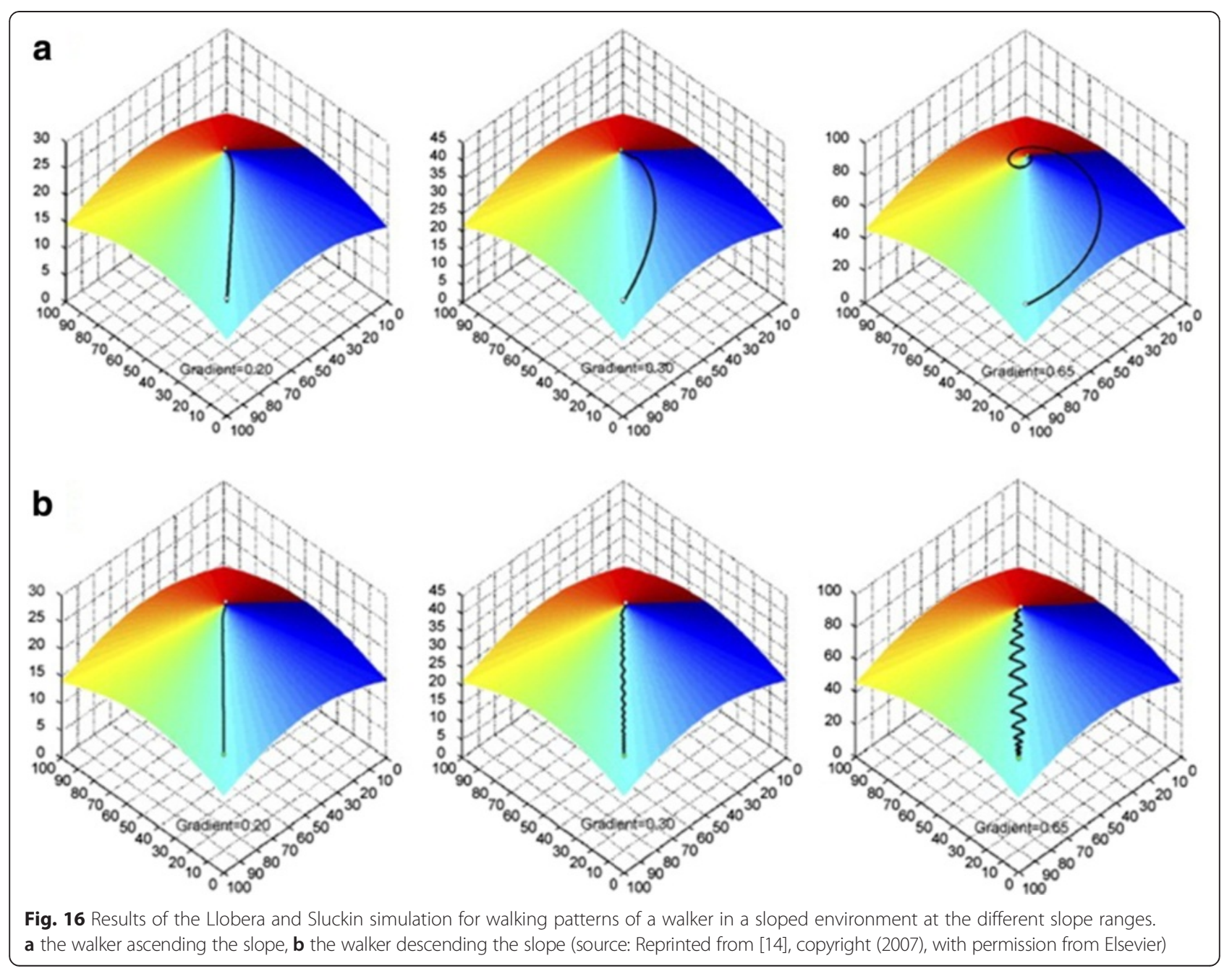


The human metabolic rate in the locomotion process is a function of speed and slope [62]. For example, Minetti et al. [59] stated that the energy cost of human locomotion on a +0.45 slope is approximately 10.5 times greater than the energy cost for walking on a flat surface at the same speed.

Furthermore, this study showed that the energy cost of human locomotion on a -0.10 slope approximately decreased to half of the energy cost needed for walking on a flat surface at the same speed. However, they found that by increasing the negative slopes, this cost is increased and the energy cost of human mobility is doubled at -0.45 compared to walking on a flat surface at the same speed.

The strategy adopted by humans for route planning in rugged terrains mainly depends on the complexity of the surface.

Thus, if the surface slope (uphill or downhill) is shallow enough, the anatomy of human body can adapt to gradient easily and compensate for the effects of the incline satisfactorily by leaning forward when walking uphill or leaning backward when walking downhill by flexing the joints in the leg.

By increasing the surface slope (positive or negative) and the transition from the shallow slope range to the semi-steep slope range, the ability of the walker to ascend or descend the surface gradually becomes more limited, and the consumption of metabolic energy for reaching the destination with the beeline path from the origin point increased dramatically. However, the physiological constraints of human anatomy dictate that the walker avoids walking on slopes steeper than the so-called critical slope threshold (for more details, see $[14,63]$ ). In this range, the human is no longer able to compensate for the incline effects by flexing the leg joints and maintaining the center of gravity over the feet.

When a pedestrian has to traverse sloped terrains, different problems emerge in the process of the optimal path selection between the origin and destination points. Insufficient human knowledge about the terrain as well as the possible obstacles and other limitations, such as steepness of the terrain, is one of the major problems that a pedestrian usually encounters during locomotion in rugged landscapes. The selection of the criterion of path optimality, such as metabolic energy, length and time of traveling or a combination of these criteria, are another notable problem in this process [49].

The walker may encounter 3 major types of inclined terrains if the surface is not flat: (1) a surface with a shallow slope, (2) a surface with a semi-steep slope range and (3) a surface with a slope equal to or greater than the critical slope threshold.

To cope with the major types of inclined terrains, the walker may adopt one of the following basic strategies (or a combination) according to several parameters, such as metabolic energy expenditure, travel time and length, comfort, safety, physical and psychological abilities, and physical condition of the sloped surfaces.

- Strategy A: To walk directly between the origin and destination on the inclined surface

The walkers usually adopt this approach where identifying an optimum solution for path selection by walking from the origin to the destination from areas with a shallow slope range to form the Euclidean shortest path (i.e., beeline path) in the Simple Topology of the Origin and Destination node. Furthermore, if the pedestrian finds this strategy to be the optimal solution for path formation in the Compound Topology of the Origin and Destination node, a BWS may emerge in the environment over time (Fig. 17).

- Strategy B: To bypass the inclined surface If the result of the rough assessment of the walker of the different available alternatives convinced him to consider the sloped surface as impassable obstacles, he will take a longer path and walk around the sloped surface instead of walking on the sloped surface to consume less energy.

- Strategy C: To choose a path on the inclined surface that is different from the direct path.

If adopting two previous options are not the optimum solution according to the constraints of the pedestrian, the walker attempts to reach the destination point by walking indirectly on the sloped surface to reach the destination by passing from the locations with less slope angles.

In terrains with steeper slopes, particularly when the walker has to walk on surfaces with a slope greater than the critical slope threshold, the optimal strategy is to walk on the inclined surface at an angle in the direction of the beeline path on the surface.

To this end, the walker changes his direction from the beeline path if he is faced with an uncompensatable gradient. The strategy that is used by the walker for reaching to the final destination can be hypnotized as follows: In the first step, he seeks a temporary destination somewhere between his current position and the final destination point on the right or left side of the beeline path (from his current location to the main destination) that can be reached directly without crossing from the areas with slopes equal to or greater than the critical slope threshold. To select the temporary destination, the walker generally considers three main points: 

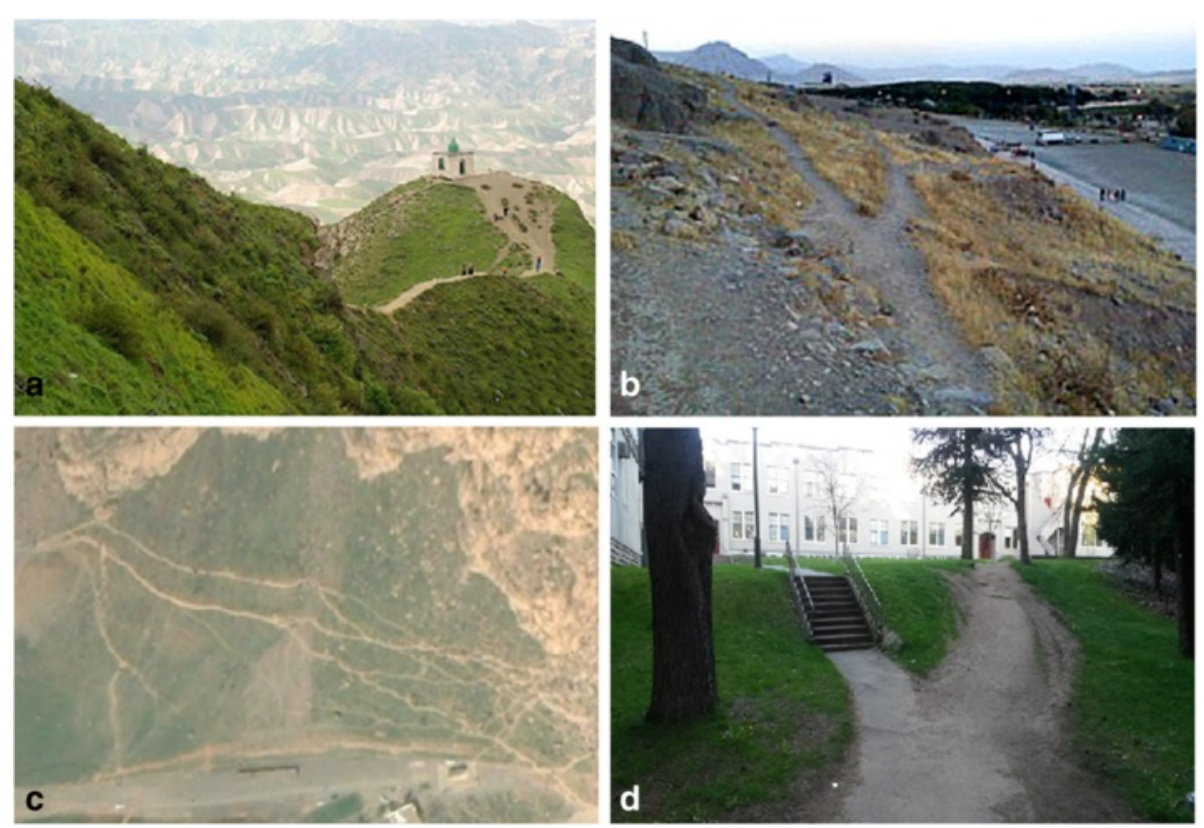

Fig. 17 Trail formation in sloped terrain as a result of the direct locomotion of pedestrians between origin and destination: a Khaled Nabi Shrine, Golestan province, Iran (source: [76]); b BWS with Y-shaped trail pattern, Mount Behistun, Kermanshah province, Iran; c Oblique satellite image of BWS with Y-shaped trail patterns, Mount Behistun, Kermanshah province, Iran (source: Google Earth); d Near Department of Geography, University of British Columbia, Vancouver, Canada

- As walking on a sloped surface is generally a very energy consuming task, after traveling for a certain distance on the sloped surface, the walker usually needs to stop to rest.

- As the walker does not like having large detours from the beeline path from the origin to the final destination, he usually prefers deviating as little as possible from the beeline path of his current location to the final destination.

- As the walker does not like to make sudden changes in the direction of his consecutive footsteps (as the sharp changes in the momentum of the walker causes discomfort, instability and changes in the walking speed), he prefers to choose the temporary point relatively far from his current location.

After reaching the temporary destination, the walker checks again whether he can reach the final destination directly. If not, he selects a new temporary destination according to the procedure that was described before and moves to that point directly. However, to compensate for the deviation from the beeline path between the origin and final destination and to correct his directional deviation, the walker, when possible, prefers to choose the next temporary point in the opposite direction of the previous direction. This procedure continues until the walker reaches the final destination point.
The abovementioned procedure and rules may cause the emergence of switchback patterns in the trails (i.e., zig-zag trails) on sloped terrains if the track is used by many walkers $[13,14]$ (Fig. 18).

A study by Minetti [63] demonstrated that for a slope of 0.25 or less, traversing the surface directly was the best path. For slope values beyond this, the optimal route was the zigzag path [64]. Another study by Leuthäusser [61] indicates that the zigzag transition occurs between the slope of 0.23 to 0.28 .

Llobera and Sluckin [14] have shown that the formation of trails with zigzag patterns corresponds to an optimization strategy that involves not only the minimization of energy (by passing from less steep gradients) but also the direction of pedestrian movement. They also discussed that, when possible, the shortcuts between the zigzag branches are taken by the pedestrians [64].

However, it should be noted that past studies show that the critical slope threshold level is different in uphill and downhill travel for the walker, so the walker may not use the same track that he selected in uphill walking when he attempts to return to the origin (for more details, see $[13,14,61,65])$.

Finally, it is necessary to mention that as different physical, psychological and social issues are usually influence the decision of the walker for path selection, the combination of these parameters may lead to different solutions in solving the path selection problem at 

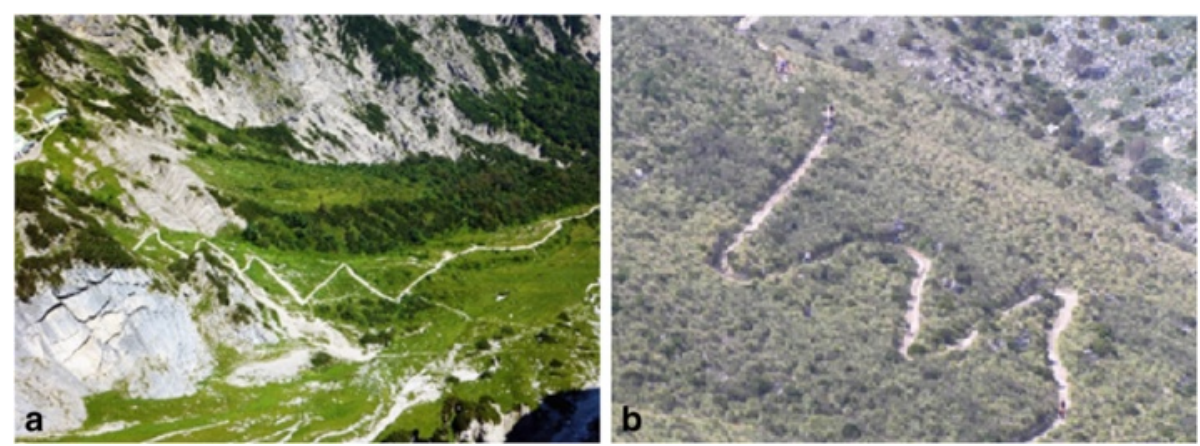

Fig. 18 Trail system in sloped terrain with switchback pattern. a Wilder Kaiser, Austria (source: Reproduced with permission from [61]); b Mallorca, Spain (source: Reprinted from [14], copyright (2007), with permission from Elsevier)

different time periods, different locations and different cultures and societies. For example, the selected path in wet seasons may be different from the selected paths in the dry seasons on the sloped areas, as walking on slippery surfaces needs more measurements. It was also observed that pedestrians from harsh environments such as mountainous areas or informal settlements often have better physical abilities and skills to walk on the sloped environments so as to pass over steeper areas during the mobility process, while normal pedestrians usually prefer to avoid such areas as much as possible.

\section{Formation of the trail system in obstructed environment}

The obstacles generally can be divided into two main categories: physical and non-physical obstacles.

A sharp local convexity and concavity of the surface (i.e., abrupt changes of the surface elevation) or any other type of environmental setting that constrains the walker's locomotion may be considered a physical obstacle where the intangible constraints for human mobility may be categorized as non-physical obstacles.

The walker determines if the obstacle is passable or impassable according to an evaluation based on his own physical abilities and consideration of some non-physical parameters.

Basically, in the case of the presence of convex or concave features of the surface (e.g., bulge, pit, clump of shrubs, fence, hedge, railing, Jersey barrier, building, etc.), the walker decides whether to consider the obstacle passable or impassable. If he considers the obstacle passable, he chooses the least metabolic energy path (i.e., Euclidean shortest path), which takes him directly over or under the obstacle (Fig. 19).

However, if he finds that he cannot pass over the obstacle, he may choose a slightly longer route that takes him around the obstacle to meet all physical and nonphysical constraints and minimize the intangible energy expenditure (cost) of the path.
In this context, a healthy and normal pedestrian considers the dimension of obstacle size scaled to his body dimensions and foot span to test whether he can remain stable when bypassing the obstacle.

Previous studies have shown that if the obstacle's height is approximately equal to or greater than the walker's lower leg length, he cannot pass over the obstacle easily. If he cannot pass under the obstacle either, then the walker tends to consider the physical obstacle impassable and will bypass it [34, 66, 67].

Other types of natural or man-made physical barriers in the environmental, such as rivers, swamps, lakes, dams, and human crowds may also be considered physical obstacles for the walker if he cannot traverse them directly.

However, if a physical obstacle blocks the participant's desired path, but the possible bypass route for the obstacle is very costly in terms of metabolic energy or time, it is observed that in practice, the walker may attempt to pass over or under the obstacle by making further measurements. For instance, if the setting of the

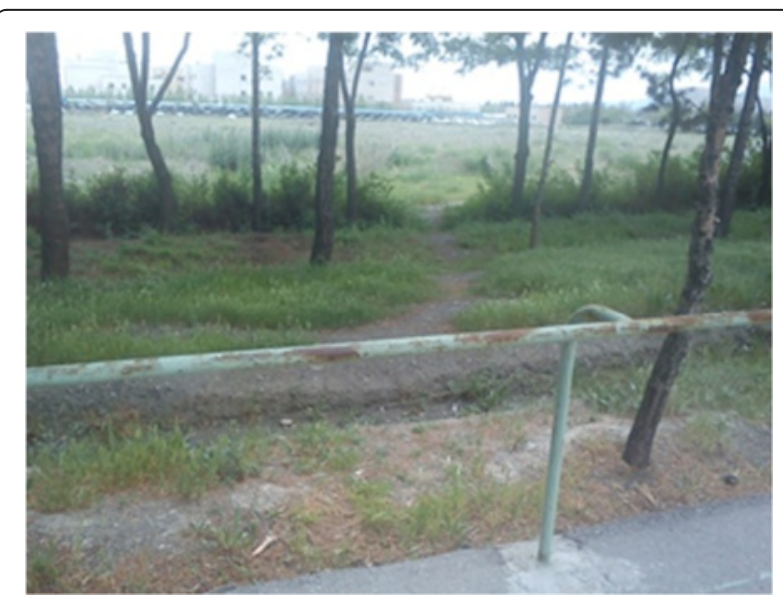

Fig. 19 A formed trail under a passable obstacle in summer, Ferdowsi University of Mashhad, Mashhad, Iran 
environment allows, he may pass the narrow water channel or muddy zone by making a bridge (Fig. 20).

When the walker encounters physical obstacles, he may consider further parameters aside from his physiological constraints to test the possibility of traversing the obstacle. As mentioned before, psychological and social parameters, such as gender, age, lifestyle, or social norms and rules may also influence the decision of walkers to pass the physical constraints directly or to go around them.

The walker may avoid traveling through some areas in the environment, even if there are no explicit physical obstacles due to non-physical parameters (e.g., gender, age, personality, lifestyle, culture or social norms) imposed that constrain him from locomotion.

For example, the walker may not pass over or under a passable railing or fence if he finding that this behavior is unacceptable in the group or society. It might be common that the residents of the informal settlements or rural areas walk on muddy surface due to their lifestyles, but the same environment may be considered impassable physical obstacles for someone from an urban area.

Some walkers may prefer to avoid walking in certain areas without enough light or during the day when the neighborhood is deserted as they may feel unsafe. Several parameters, such as gender, age, personality of the walker and background of the neighborhood in the perspective of the society and mentality of the walker may play a role in making this decision.

Another good example of these types of non-physical obstacles in the context of informal settlements might be observed in some informal African settlements. In a typical Swahili housing pattern, the land owners usually construct two buildings in a plots (a larger building as the

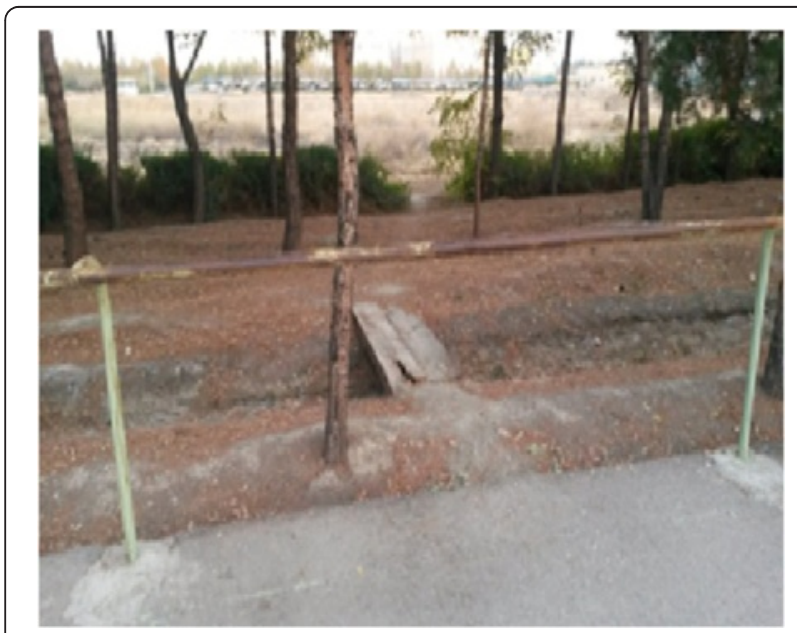

Fig. $20 \mathrm{~A}$ formed trail under a passable obstacle in fall, Ferdowsi University of Mashhad, Mashhad, Iran (same location as Fig. 19). The pedestrians who used the trail made a bridge from used lumber to pass over the muddy stream main building and a smaller building as an extension) and the boundary of the plot may not be surrounded by any physical barriers. However, in practice, it is observed that the settlement residents do not pass from the space between these large and small building and deviate from their path to bypass this intangible barrier as they recognized the land tenure and privacy of the owner as the result of the social agreements.

If the walker respects social rules according to his personality and walking on urban green spaces is not allowed in that society, the walker usually does not pass over the lawn (even if there is no barrier to protect it) and will attempt to bypass it by going around the lawn.

The previous study by Warren et al. [68] shows that in the case of impassable obstacles, the pedestrian makes gradual turns when walking in the environment by changing his heading direction over several steps rather than abrupt switching direction. Furthermore, they found that pedestrians do not walk in a continuous arc to the destination but turn onto a linear segment that aligns the heading direction with the destination. They also stated that the rate of turning is dependent on both the distance to the destination or the obstacle and its respective angle with respect to the heading direction. They concluded that the turning of the pedestrian is not a biomechanically stereotyped action but is controlled by the information for the observer's locomotion relative to objects and obstacles in the scene.

At the end of this section, it is necessary to note the temporal aspect of the presence of the obstacles. When most obstacles have static natures in the environment, some obstacles have dynamic natures. For example, seasonal rivers or swamps or temporary threats in nature may be considered obstacles at a certain period of time and causes the walker to avoid certain locations in the environment. However, by removing these temporary constraints in the environment, the walker may consider those areas as potential areas for travel. Such temporary barriers may influence the existence of the trail or spatial structure of the formed trail system in the environment over time.

Formation of trail system in a non-uniform environment In an even and obstacle-free environment, the walker may prefer some areas over the others for travel if the environment is not uniform from his point of view.

The simplest example of this type of preference for pedestrians was noted in the previous sections. In this sense, with the emergence of well-trodden tracks in the environment, individuals prefer to use these over nonconsolidated surfaces in nature.

The different physical, social or physiological parameters determine the favor and disfavor of the walker with regard to the different spots in the environment. 
As was discussed before, in a more complex environment, the pedestrian considers various parameters in a multi-criteria decision making process to maximize his benefits and fulfill his desires in the process of informal path formation. In this sense, he may detour from the beeline path if some spots around the walker attract him significantly or areas in the environment are unfavorable to him.

The physical characteristics of the walking surface are important elements for an individual in the process of path selection in the outdoor environment. Pedestrians usually prefers stiff, dense and smooth surfaces over loose, soft and uneven bumpy surfaces as they can support their weight during the mobility process and provide ease of travel at a normal speed. The Lejeune et al. [69] study shows that walking on loose-soil surfaces such as sand requires 2.1-2.7 times more human energy expenditure than walking on solid ground at the same speed. Furthermore, Haisman and Goldman [70] stated that the human energy expenditure while walking on difficult terrains and thick bushes may overwhelm human physiological limits in comparison to walking on the smooth surfaces. Walking on rough, uneven and bumpy surfaces requires more energy than walking on a smooth surface. The previous study showed that the cost of walking over rough and bumpy surfaces, such as ploughed fields, is $50 \%$ greater than walking on a smooth surface [64].

Thus, walkers usually prefer stiff, smooth surfaces as much as possible as they can walk with less physical effort and at greater speeds. If part of the beeline path stretches on a loose surface and the walker finds a stiff surface in his vicinity, he may deviate from the direct path if the result of his evaluation confirms that this detour is an optimal solution.

The unpleasant smell of a garbage depot or a marsh may force the walker to consider changing his route. Furthermore, the pleasant shadow of trees during a hot summer near a direct path may encourage the walker to make a detour from the beeline path to increase his comfort.

Finally, it is necessary to note that in the process of evaluating the environment, the extreme like or dislike of the pedestrian leads to the total omission of some spots in the spatial decision making of the individual.

If the walker likes an area, he considers these areas to be high value regions and neglect other spots in the environment. Furthermore, if he does not wish to travel through some areas in the environment, he may encounter those areas as obstacles and avoid them.

\section{Lifetime of informal infrastructure}

In addition to spatial characteristics of human trail systems, as mentioned, the temporal dimension of human trail formation is another notable characteristic of the trail formation phenomenon. So far, the different spatiotemporal aspects of informal infrastructures have been reviewed and discussed in the previous sections. However, to gain better insight into the temporal aspects of the informal infrastructure formation phenomenon, in this section, other issues on the life span of informal infrastructures will be discussed and reviewed in more detail.

As discussed before, the informal infrastructure is an adaptive system, and a trail system is able to respond to changes that happen in the system. This type of change in the system may fully or partially change the spatial pattern of the trail or even may affect the existence of the trail system in the environment.

The life span and durability of the informal infrastructure is the function of the number of pedestrians who use the path over time. Consequently, human footprints and formed trails in an open space can fade or disappear if the track or trail is abandoned for a long period of time or is blocked and is not used by the pedestrians frequently due to different physical, social or economic factors. In other words, as the rate of surface weathering and decay or vegetation growth exceeds the rate of surface damage, compaction and trampling, the track or trail will gradually vanish over time.

For example, the formed beeline paths in the compound topology of the origin and destination points are gradually abandoned (fully or partially) over time as the DWS transitions to the BWS and the emergence of the compromise trail system. The decrease in the attractiveness of the destination or the security or safety conditions of the neighborhood, season and trail's surface condition or construction of a formal paved path to the destination are other examples of parameters that may temporarily or permanently affect the life span and existence of the trail in the environment.

A new obstacle or barrier to the system may change the equilibrium of the system and affect the existence of the trail fully or partially.

For instance, in an informal settlement, a new building may be built in the path of an informal track and block the path if the trail is used only by a few dwellers and is not consolidated well in the settlement. Thus, if enough space is available around the newly constructed building, users bypass the new obstacle, and a new track is formed gradually around the new building. However, even though this sort of paths can survive for a la period of time, past studies showed that most of these trails will disappear during the middle stages of the extension stage and later during the densification (infilling) stage of the informal settlement dynamic (for more details, see [7]).

Furthermore, covering or blocking the formed trail (fully or partially) with natural or man-made obstacles such as seasonal floods, swamps, or dams may cause abandonment of the trail (fully or partially) temporarily or permanently. 


\section{Conclusion}

In this paper, some important spatio-temporal aspects of the informal infrastructure formation phenomenon that are necessary for developing a conceptual model of this phenomenon have been reviewed and studied. Reviewing the limited existing literature on this area shows that this topic has not yet received much attention, and further independent studies are needed to clarify the different aspects of the informal infrastructure growth mechanisms. Furthermore, most of the aforementioned studies have only focused on developing ad-hoc parameters and rules and were mainly developed according to theoretical backgrounds such as mathematics, physics or bionics-inspired methodologies without much study of human physical, psychological and cognitive behaviors. This study showed that the majority of previous studies in this field were mainly focused on studying and modeling abstract case studies without any precedent in the real world. However, even the few existing studies that aimed to study and model the realistic informal infrastructure only included simplified systems, and the model often cannot fully reflect the dynamics and patterns in the real world. Thus, the morphology of the simulated patterns cannot be validated quantitatively. In this review and study, we have offered an overview of some of the most important driving factors and aspects of informal infrastructure formation that are needed to build the theoretical background for developing spatially explicit, cognitively plausible conceptual models for future study. In this context, we focused on understanding, reviewing and discussing previous studies on the formation of the informal infrastructure system in the Simple and Compound Topology of origin and destination node(s) as well as the emergent large-scale, self-organized patterns in system. Considering a flat, obstacle-free and uniform environment in the first step, we focused on the informal infrastructure formation on complex terrains. Finally, the life span of the informal infrastructure was briefly discussed to summarize and identify the open and noteworthy issues for future study. Overall, as the understanding of informal infrastructure growth phenomena may help us plan, conserve and manage open spaces in planned and unplanned environments and enable us to predict and manage informal settlement growth in a more sophisticated way, future study to understand the different aspects of this phenomenon to develop more holistic and realistic conceptual models in this area seems necessary.

One of the main challenges in the modeling and simulation of trail formation dynamic is the lack of human empirical data on the different behaviors and dynamics of the system and absence of enough information on the different stages and patterns of the physical evolution in the system. To understand the underlying mechanisms, create plausible scenarios for informal infrastructure formation phenomenon and for better evaluation and calibration of the developed models, it is essential to acquire the systematic spatio-temporal knowledge on this phenomenon. In this sense, the spatio-temporal analysis of the geo-spatial data that was collected from the technical sensors (i.e., remote sensing data and in-situ sensor data) and human sensors (i.e., human-generated data, such as social media data) at multiple scales and times can help the researchers to gain a deeper understanding from the informal infrastructure formation phenomenon and develop more plausible models.

Last, the existing studies in the area of studying the informal infrastructure system only concentrated on the process of trail system formation and modeling of pedestrian behaviors in the system. Insufficient attention has been paid to the travel forecasting process in the informal infrastructure. Therefore, studies in the area of trip generation (studying the role of social and economic attributes of pedestrians in trip generation) and destination choice (i.e., trip distribution) is required. This study may enable researchers and industry practitioners to understand who moves in the environment, when trips are planned, and where they go, that consequently help them forecast and model the specific trip behaviors and daily activities of pedestrians as well as pedestrians' trips, schedule, trip sequences, trip patterns and the origin and destination of trips to develop a comprehensive transportation model for the simulation of informal infrastructure formation.

\section{Acknowledgements \\ The authors would like to thank the people and publishers who kindly allowed permission for the reproduction of their figures.}

\section{Authors' contributions}

$\mathrm{HV}$ conceived of the study and carried out design, conception, review of studies, and analysis, categorizing and interpretation of data and drafted the manuscript. WY participated in coordination of the study and helped to review the manuscript. Both authors read and approved the final manuscript.

\section{Competing interests}

The authors declare that they have no competing interests.

\section{Author details}

${ }^{1}$ EcoGIS Lab, Graduate School of Media and Governance, Shonan Fujisawa Campus, Keio University, Fujisawa, Japan. ' Lab for Advanced Spatial Analysis, University of British Columbia, Vancouver, Canada. ${ }^{3}$ Faculty of Environmental and Information Studies, Shonan Fujisawa Campus, Keio University, Fujisawa, Japan.

Received: 29 January 2016 Accepted: 19 July 2016

Published online: 30 July 2016

\section{References}

1. UN-Habitat. Global Report on Human Settlements 2003: The Challenge of Slums. Sterling, VA: Earthscan; 2003

2. Sietchiping R. Prospective slum policies: conceptualization and implementation of a proposed informal settlement growth model. In: Third Urban Research Symposium"Land, Urban and Poverty Reduction. 2005. p. 4-6.

3. Throgmorton J, Eckstein B. Desire Lines: The Chicago Area Transportation Study and the Paradox of Self in Post-War America. Literary and Visual Representations of Three American Cities, Desire Lines: The Chicago Area 
Transportation Study and the Paradox of Self in Post-War America. Literary and Visual Representations of Three American Cities, 1870s to 1930. In: A project web site of the 3 Cities Project of the Universities of Nottingham and Birmingham, United Kingdom. 2000.

4. Gallagher P. Creating a Pedestrian Level-of-Service Index for Transit Stops: Evidence from Denver's Light Rail System (2012). Master's Theses. Paper 268, http://digitalcommons.uconn.edu/gs_theses/268.

5. Gallagher P, Marshall W, Atkinson-Palombo C. Missing Links. Transportation Research Record: Journal of the Transportation Research Board. 2013;2393:59-65.

6. Liddle MJ. A selective review of the ecological effects of human trampling on natural ecosystems. Biological Conservation. 1975;7(1):17-36.

7. Vahidi H, Yan W. Towards Spatially Explicit Agent-based Model for Simulation of Informal Transport Infrastructure Indirect Growth Dynamic in Informal Settlements. ISPRS-International Archives of the Photogrammetry, Remote Sensing and Spatial Information Sciences. 2014;1:273-9.

8. Dridi MH. Pedestrian flow simulation validation and verification techniques, arXiv preprint arXiv:14100603. 2014.

9. Bonneaud S, Warren WH. A behavioral dynamics approach to modeling realistic pedestrian behavior. Heidelberg: Pedestrian and Evacuation Dynamics Springer; 2012.

10. Hofmann P, Taubenbock H, Werthmann C. Monitoring and modelling of informal settlements - A review on recent developments and challenges. In: Urban Remote Sensing Event (JURSE), 2015 Joint: March 30 2015-April 1 2015. 2015. p. 1-4.

11. Vahidi H, Mobasheri A, Yan W. Looking for Human Footprint in Nature: Collaborative Mapping for Monitoring of Self-organized Patterns in Human Trail Systems. In: The 27th International Cartographic Conference (ICC 2015): 2015; Rio de Janeiro, Brazil.

12. Helbing D, Keltsch J, Molnar P. Modelling the evolution of human trail systems. Nature. 1997;388(6637):47-50.

13. GILKS SJ, HAGUE JP. Mountain trail formation and the active walker model. International Journal of Modern Physics C. 2009;20(06):869-90.

14. Llobera M, Sluckin TJ. Zigzagging: Theoretical insights on climbing strategies. Journal of Theoretical Biology. 2007;249(2):206-17.

15. Burns $M$, Weaver A. Exploring sustainability science: A Southern African perspective: African Sun Media; Stellenbosch 7600, South Africa. 2008. http://www.africansunmedia.co.za/Portals/0/files/extracts/Exploring\%20 Sustainability\%20Science\%20Extract.pdf.

16. Wilson AG. Complex spatial systems: Challenges for modellers. Mathematical and Computer Modelling. 2002;36(3):379-87.

17. Paczuski M, Bak P. Self-organization of complex systems, arXiv preprint cond-mat/9906077. 1999

18. Moussaid M, Garnier S, Theraulaz G, Helbing D. Collective Information Processing and Pattern Formation in Swarms, Flocks, and Crowds. Topics in Cognitive Science. 2009; 1(3):469-97

19. Bonabeau E, Theraulaz G, Deneubourg J-L, Aron S, Camazine S. Selforganization in social insects. Trends in Ecology \& Evolution. 1997; 12(5):188-93.

20. Couzin ID, Krause J. Self-Organization and Collective Behavior in Vertebrates. In: Advances in the Study of Behavior. vol. Volume 32: Academic Press; United States. 2003;1-75. http://www.sciencedirect.com/science/article/pii/ S0065345403010015

21. Cialdini RB, Goldstein NJ. Social Influence: Compliance and Conformity. Annual Review of Psychology. 2004;55(1):591-621.

22. Goldstone RL, Jones A, Roberts ME. Group path formation. Systems, Man and Cybernetics, Part A: Systems and Humans, IEEE Transactions on. 2006;36(3):611-20

23. Latané B, Bourgeois MJ. Experimental Evidence for Dynamic Social Impact: The Emergence of Subcultures in Electronic Groups. Journal of Communication. 1996:46(4):35-47.

24. Parks CD, Sanna LJ. Group performance and interaction: Westview Press: Boulder, Colorado. 1999

25. Sherif M. A study of some social factors in perception. Archives of Psychology (Columbia University) 1935;27(187):17-22.

26. Rogers EM. Diffusion of innovations: Simon and Schuster; New York City, New York, United States. 2010. http://books.simonandschuster.com/ Diffusion-of-Innovations-4th-Edition/Everett-M-Rogers/9781451602470.

27. Rogers EM. Elements of diffusion. Diffusion of innovations. 2003;5:1-38.

28. Valente TW. Social network thresholds in the diffusion of innovations. Social Networks. 1996;18(1):69-89.
29. Goldstone RL, Gureckis TM. Collective Behavior. Topics in Cognitive Science. 2009;1(3):412-38.

30. Dorigo M, Bonabeau E, Theraulaz G. Ant algorithms and stigmergy. Future Generation Computer Systems. 2000;16(8):851-71.

31. Wolbers T, Hegarty M. What determines our navigational abilities? Trends in Cognitive Sciences. 2010;14(3):138-46.

32. Onatmiiu E, Slauh S. A Computational Model for Simulation of Human Navigation Behaviour. In: STAIRS 2004: Proceedings of the Second Starting Al Researchers' Symposium: 2004. IOS Press: Amsterdam, Netherlands. 84.

33. Gifford R. Environmental psychology: Principles and practice. Colville, WA: Optimal books; 2007.

34. Patla AE. Understanding the roles of vision in the control of human locomotion. Gait \& Posture. 1997;5(1):54-69.

35. Warren W, Fajen B. Behavioral Dynamics of Visually Guided Locomotion. In: Fuchs A, Jirsa V, editors. Coordination: Neural, Behavioral and Social Dynamics. Berlin Heidelberg: Springer; 2008. p. 45-75.

36. Raubal M, Egenhofer MJ. Comparing the complexity of wayfinding tasks in built environments. Environment and planning B. 1998;25:895-914.

37. Passini R. Spatial representations, a wayfinding perspective. Journal of Environmental Psychology. 1984;4(2):153-64.

38. Rovine MJ, Weisman GD. Sketch-map variables as predictors of way-finding performance. Journal of Environmental Psychology. 1989;9(3):217-32.

39. Lin J-H, Ho C-H, Ngan K-L, Tu J-H, Weerapaiboon W. The Effects of Senses of Direction on Wayfinding Behaviors: Evidence from Biking Tourists. SHS Web of Conferences. 2014;12:01001.

40. Antonini G. A discrete choice modeling framework for pedestrian walking behavior with application to human tracking in video sequences. EPFL; Switzerland. 2005. https://infoscience.epfl.ch/record/55416.

41. Giannopoulos I, Kiefer P, Raubal M, Richter K-F, Thrash T. Wayfinding Decision Situations: A Conceptual Model and Evaluation. In: Geographic Information Science. Edited by Duckham M, Pebesma E, Stewart K, Frank A, vol. 8728: Springer International Publishing; 2014: 221-234. http://www. springer.com/us/book/9783319115924.

42. Contreras F, Farjas M. Theory and Practice of Cost Functions. In: 38th Annual Conference on Computer Applications and Quantitative Methods in Archaeology: 2010; Granada, Spain.

43. Llobera M. Understanding movement: a pilot model towards the sociology of movement. NATO ASI SERIES A LIFE SCIENCES. 2000;321:65-84.

44. Givoni B, Goldman RF. Predicting metabolic energy cost. Journal of Applied Physiology. 1971;30(3):429-33.

45. Margaria R, Cerretelli P, Aghemo P, Sassi G. Energy cost of running. Journal of Applied Physiology. 1963;18(2):367-70.

46. Kay A. Route Choice in Hilly Terrain. Geographical Analysis. 2012;44(2):87-108.

47. Pandolf KB, Haisman MF, Goldman RF. Metabolic Energy Expenditure and Terrain Coefficients for Walking on Snow. Ergonomics. 1976;19(6):683-90.

48. Soule RG, Goldman RF. Terrain coefficients for energy cost prediction. Journal of Applied Physiology. 1972;32(5):706-8.

49. Jaklič G, Kanduč T, Praprotnik S, Žagar E. Energy Minimizing Mountain Ascent. J Optim Theory Appl. 2012;155(2):680-93.

50. Balstrøm T. On identifying the most time-saving walking route in a trackless mountainous terrain. Geografisk Tidsskrift-Danish Journal of Geography. 2002:102(1):51-8.

51. Minetti AE, Alexander RM. A Theory of Metabolic Costs for Bipedal Gaits. Journal of Theoretical Biology. 1997;186(4):467-76.

52. Simon HA. A behavioral model of rational choice. The quarterly journal of economics 1955;69(1):99-118.

53. Helbing D, Schweitzer F, Keltsch J, Molnár P. Active walker model for the formation of human and animal trail systems. Physical Review E. 1997:56(3):2527-39.

54. Helbing $D$, Molnár P, Schweitzer F. Computer simulations of pedestrian dynamics and trail formation, arXiv preprint cond-mat/9805074. 1998.

55. Goldstone RL, Roberts ME. Self-organized trail systems in groups of humans. Complexity. 2006;11(6):43-50.

56. Crescenzi P, Kann V. A compendium of NP optimization problems. URL: http://www.nada.kth.se/ viggo/problemlist/compendium.html 1997.

57. Crescenzi P, Kann V, Halldórsson MM, Karpinski M, Woeginger G. A compendium of NP optimization problems, 2005. URL http://www.nada.kth. se/ viggo/problemlist/compendium.html 2005.

58. Gander MJ, Santugini K, Steiner A. Shortest road network connecting cities. Bollettino dei docenti di matematica. 2008;56:9-19. 
59. Minetti AE, Moia C, Roi GS, Susta D, Ferretti G. Energy cost of walking and running at extreme uphill and downhill slopes. Journal of Applied Physiology. 2002;93(3):1039-46.

60. Rees WG. Least-cost paths in mountainous terrain. Computers \& Geosciences. 2004;30(3):203-9.

61. Leuthäusser U. About walking uphill: time required, energy consumption and the zigzag transition [http://www.sigmadewe.com/fileadmin/user upload/pdf-Dateien/Bergaufgehen_engl.pdf]

62. Sparks FT, O'Reilly KC, Kubie JL. What is optimized in an optimal path? Behavioral and Brain Sciences. 2013;36(05):566-6.

63. Minetti AE. Optimum gradient of mountain paths. Journal of Applied Physiology. 1995;79(5):1698-703.

64. Murrieta-Flores P. Travelling in a prehistoric landscape: Exploring the influences that shaped human movement. In: Making History Interactive Computer Applications and Quantitative Methods in Archaeology (CAA)[Proceedings of the 37th International Conference, Williamsburg, Virginia, United States of America. 2009. p. 258-76.

65. Alexander RM. Energetics and optimization of human walking and running: the 2000 Raymond Pearl memorial lecture. American Journal of Human Biology. 2002;14(5):641-8.

66. Patla $A E$, Rietdyk $S$. Visual control of limb trajectory over obstacles during locomotion: effect of obstacle height and width. Gait \& Posture. 1993; (1):45-60

67. Warren Jr WH, Young DS, Lee DN. Visual control of step length during running over irregular terrain. Journal of Experimental Psychology: Human Perception and Performance. 1986:12(3):259.

68. Warren W, Fajen B, Belcher D. Behavioral dynamics of steering, obstacle avoidance, and route selection. Journal of Vision. 2001;1(3):184-4.

69. Lejeune TM, Willems PA, Heglund NC. Mechanics and energetics of human locomotion on sand. Journal of Experimental Biology. 1998;201(13):2071-80.

70. Haisman M, Goldman R. Effect of terrain on the energy cost of walking with back loads and handcart loads. Journal of applied physiology. 1974:36(5):545-8.

71. Schindler P. Shanty town outside Cape Town. [http://peterschindler. blogspot.jp/2009/11/premier-classe-and-cape-town-my.html]; 2009.

72. Green D. Flying toilets, mobile banking and the stress-free mini hotel: Kibera in photos. [http://www.oxfamblogs.org/fp2p/?attachment_id=8275]; 2012

73. Hendriksen H. South Africa Travel Photography [https://www.flickr.com/ photos/hanshendriksen/6964594624]; 2012.

74. Sotoudeh Shayegh P. Climbing Darabad Mount [http://neshate-koohestan. blogfa.com/post/1937]

75. Diffusion of Ideas [https://commons.wikimedia.org/wiki/File:Diffusion_ of_ideas.svg]

76. Khaled Nabi Shrine, Relaxing and Mysterious [http://www.mashreghnews.ir/ fa/news/374751/\%D8\%B9\%DA\%A9\%D8\%B3-\%D8\%B2\%DB\% 8C\%D8\%A7\%D8\%B1\%D8\%AA\%DA\%AF\%D8\%A7\%D9\%87-\%D8\%AE\% D8\%A7\%D9\%84\%D8\%AF\%D9\%86\%D8\%A8\%DB\%8C\%D8\%A2\%D8\%B1\% D8\%A7\%D9\%85\%D8\%B4\%E2\%80\%8C\%D8\%A8\%D8\%AE\%D8\%B4-\%D9\%88\%D8\%B1\%D8\%A7\%D8\%B2\%D8\%A2\%D9\%84\%D9\%88\%D8\%AF]

77. Deji OF. Gender and Rural Development: Introduction, vol. 2. Münster: LIT Verlag; 2011

78. Rajagopal: Architecting enterprise: managing innovation, technology, and global competitiveness: Palgrave Macmillan; 2014.

79. Lee $\mathrm{Y}-\mathrm{H}, \mathrm{Hsieh} \mathrm{Y}-\mathrm{C}$, Hsu C-N. Adding innovation diffusion theory to the technology acceptance model: Supporting employees' intentions to use e-learning systems. Journal of Educational Technology \& Society. 2011;14(4):124-37. http://www.ifets.info/.

80. Interim services for informal settlements. [http://www.specifile.co.za/ specifier/housing-and-human-settlements/890-interimservices-for-informalsettlements]; 2011

\section{Submit your manuscript to a SpringerOpen ${ }^{\circ}$ journal and benefit from:}

- Convenient online submission

- Rigorous peer review

- Immediate publication on acceptance

- Open access: articles freely available online

- High visibility within the field

- Retaining the copyright to your article

Submit your next manuscript at $\gg$ springeropen.com 